TRANSACTIONS OF THE

AMERICAN MATHEMATICAL SOCIETY

Volume 362, Number 9, September 2010, Pages 4511-4536

S 0002-9947(10)04807-5

Article electronically published on April 14, 2010

\title{
HÖLDER ESTIMATES FOR DEGENERATE ELLIPTIC EQUATIONS WITH COERCIVE HAMILTONIANS
}

\author{
I. CAPUZZO DOLCETTA, F. LEONI, AND A. PORRETTA
}

\begin{abstract}
We prove a priori estimates and regularity results for some quasilinear degenerate elliptic equations arising in optimal stochastic control problems. Our main results show that strong coerciveness of gradient terms forces bounded viscosity subsolutions to be globally Hölder continuous, and solutions to be locally Lipschitz continuous. We also give an existence result for the associated Dirichlet problem.
\end{abstract}

\section{INTRODUCTION}

In this work we consider a class of second order degenerate elliptic equations with superlinear Hamiltonians, whose simplest model is the following:

$$
-\operatorname{tr}\left(A(x) D^{2} u\right)+\lambda u+|D u|^{p}=f(x), \quad x \in \Omega .
$$

Here $\Omega$ is an open bounded subset of $\mathbf{R}^{N}, N \geq 2$, and $A: \Omega \mapsto \mathcal{S}_{N}^{+}$is a bounded and continuous map into the space of symmetric nonnegative matrices of order $N$, $\lambda \geq 0$ and $p>1$ are given numbers and $f: \Omega \mapsto \mathbf{R}$ is a continuous function.

It is well known that equations such as (1.1) arise in stochastic control as the Hamilton-Jacobi-Bellman equation satisfied by the value function of optimization problems for degenerate diffusion processes. More precisely, let $X_{t}$ be the state of the controlled system governed by the Ito differential equation

$$
d X_{t}=a\left(X_{t}\right) d t+\sqrt{2} \Sigma\left(X_{t}\right) d W_{t}, \quad X_{0}=x \in \Omega,
$$

where $W_{t}$ is an $M$-dimensional Brownian motion, $\Sigma(x)$ is a nonnegative $N \times M$ matrix and the drift $a\left(X_{t}\right)$ is interpreted as a feedback control, and consider the cost functional

$$
J(x, a)=E_{x}\left\{\int_{0}^{\tau_{x}}\left[f\left(X_{t}\right)+\frac{p-1}{p^{\frac{p}{p-1}}}\left|a\left(X_{t}\right)\right|^{\frac{p}{p-1}}\right] \mathrm{e}^{-\lambda t} d t+\varphi\left(X_{\tau_{x}}\right) \mathrm{e}^{-\lambda \tau_{x}}\right\},
$$

where $E_{x}$ is the conditional expectation with respect to $X_{0}=x, \tau_{x}$ is the first exit time from $\Omega$ and $a$ belongs to a set $\mathcal{A}_{x}$ of admissible control laws. Then, classical Dynamic Programming arguments, see [9], show that the value function

$$
u(x)=: \inf _{a \in \mathcal{A}_{x}} J(x, a)
$$

Received by the editors January 28, 2008.

2010 Mathematics Subject Classification. Primary 35J70, 35B65.

Key words and phrases. Degenerate elliptic equations, coercive Hamiltonians, Hölder regularity, viscosity (sub)solutions.

This work was partially supported by PRIN-COFIN 2005 Project "Viscosity, metric and control theoretic methods in nonlinear partial differential equations".

(C)2010 American Mathematical Society 
is a viscosity solution of equation (1.1) with $A(x)=\Sigma(x) \Sigma(x)^{T}$. In addition, the equation can be complemented either with the Dirichlet boundary condition $u=\varphi$ (for the so-called exit-time problem, $\varphi$ representing a cost to be paid when the state reaches the boundary) or with the condition that $\tau_{x}=+\infty$; i.e., the process stays in $\Omega$ for all time with probability one (the state constraint problem).

Several contributions exist in the literature for similar problems. In the case of equation (1.1), we refer in particular to [10, for a deep and complete study of the state constraint problem in the model nondegenerate case $A(x) \equiv I d$ (purely Brownian motion). In that situation, the authors prove that the value function $u$ is the maximal solution of (1.1), that it is locally Lipschitz and that, if $1<p \leq 2$, then $u$ blows up at the boundary, while if $p>2, u$ is bounded and Hölder continuous up to the boundary. This fundamental difference between the cases $p \leq 2$ and $p>2$ reflects a similar feature occurring in the study of the exit-time problem (see [4]); namely, if $1<p \leq 2$, then the Dirichlet problem can be solved (in the classical sense) for any boundary datum $\varphi$, while if $p>2$, this is no longer true and there can be a loss of boundary conditions. In that case, the best one can expect in general is that the Dirichlet condition $u=\varphi$ is satisfied in a generalized relaxed sense.

When studying similar problems (involving state constraint or relaxed boundary conditions), a crucial role is played by gradient estimates and local barrier effects. Motivated by this, as well as by related applications to corresponding ergodic (or homogenization) problems, we study here the regularity properties of (viscosity) solutions of (1.1) in the general possibly degenerate case $(A(x) \geq 0)$ with special attention to estimates independent of the boundary behavior of the solutions. A crucial role is played by the superlinear character of the Hamiltonian in order to get general Hölder or Lipschitz estimates. Note that when (1.1) is the Hamilton-JacobiBellman equation associated to the control problem (1.2), then standard synthesis procedures show that the vector field $a(x)=-p|D u(x)|^{p-2} D u(x)$ provides an optimal feedback law and hence a further motivation in investigating the Lipschitz regularity of solutions.

The main results that we give concern the superquadratic case $p>2$, where we prove that viscosity subsolutions of (1.1) are Hölder continuous. In a simple version, this result can be stated as follows:

Theorem 1.1. Let $\Omega \subset \mathbf{R}^{N}$ have Lipschitz boundary and satisfy the uniform interior sphere condition. If $u$ is a bounded, upper semicontinuous, viscosity subsolution of (1.1) with $p>2$, then $u$ is Hölder continuous in $\bar{\Omega}$ and satisfies

$$
|u(x)-u(y)| \leq M|x-y|^{\alpha} \quad \forall x, y \in \bar{\Omega}, \alpha=\frac{p-2}{p-1},
$$

where $M$ depends on $p,\|A\|_{L^{\infty}(\Omega)},\|f\|_{L^{\infty}(\Omega)},\left\|\lambda u^{-}\right\|_{L^{\infty}(\Omega)}$ and on $\partial \Omega$.

The above result extends the Hölder estimates of classical solutions, proved in 10] for the model case $A(x)=I d$. What is remarkable is that the result of Theorem 1.1 applies not only to solutions but even to subsolutions, which is not common for second order problems. This reminds us instead of what happens for coercive first order equations, where subsolutions are known to be Lipschitz continuous (see e.g. 1], 2], 12]). Here, when $p>2$, the strongly coercive first order term in (1.1) allows us to control the possible oscillations due to the diffusion term. Moreover, if $A(x) \equiv 0$, our proof shows that the exponent $\alpha$ can be taken to be one, which 
is consistent with the first order case. Otherwise, the exponent $\alpha$ found in (1.3) is optimal.

Another interesting feature of the above estimate is that it is independent of the boundary value of the solution $u$. This means that in the study of the Dirichlet problem the Hölder regularity of the boundary datum $\varphi$ is necessary in order to find a strong solution, satisfying pointwise the condition $u=\varphi$. This allows us to complement previous results obtained in 4 concerning the relaxed formulation of the Dirichlet problem associated to (1.1), in case $A(x)$ does not degenerate in the normal direction at $\partial \Omega$. More precisely, under some compatibility conditions between $f$ and $\varphi$ and assuming an upper bound on the Hölder constant of $\varphi$, we prove (Theorem 2.12) the existence of a $C^{0, \frac{p-2}{p-1}}(\bar{\Omega})$ viscosity solution of (1.1) satisfying pointwise the Dirichlet condition $u=\varphi$.

The above results, which hold in the range $p>2$, will be proved in Section 2. More precisely: in Section 2.1 we prove the basic local Hölder estimate for subsolutions of (1.1); this actually contains the main idea of our proofs and admits several extensions with respect to the model problem (1.1) (see also Theorem 2.11). In Section 2.2 we extend the local estimate up to the boundary by using only the regularity and geometric properties of $\partial \Omega$, thus obtaining the complete result (Theorem 2.7). In Section 2.3 we consider, under this new light, the Dirichlet problem associated to (1.1).

On the other hand, in Section 3 we address the issue of Lipschitz continuity for solutions, and this for the whole superlinear range $p>1$. Thus we prove in Theorem 3.1 that viscosity solutions of (1.1) are locally Lipschitz continuous and

that the gradient estimate $|D u(x)| \leq \frac{K}{d(x)^{\frac{1}{p-1}}}$ holds almost everywhere in $\Omega$, where $d(x)$ is the distance function from the boundary of $\Omega$. Theorem 3.1 extends previous similar results obtained using the classical Bernstein method in [10, [13] for the nondegenerate case or in the recent paper 14 for the degenerate problem set in the whole space. Similar results have been established in 3 through the so-called weak Bernstein method applied to viscosity solutions. Let us point out with respect to these previous works that our proof here uses a different approach which does not require regularization procedures and avoids, in any sense, the differentiation of the equation, applying directly to continuous viscosity solutions. A similar approach is possible by using in its full strength the coercivity of the Hamiltonian. Moreover, though at the expense of a (quite hard) localization of our test functions, we obtain an estimate which is independent of the boundary behavior and is stable as $\lambda \rightarrow 0$, being applicable to ergodic as well as to homogenization problems.

\section{HÖLDER CONTINUity OF SUBSOlutions}

Let $\Omega \subset \mathbf{R}^{N}(N \geq 2)$ be an open and bounded set. We consider a function $u$ which satisfies in the standard viscosity sense (see [8]) the inequality

$$
-\operatorname{tr}\left(A(x) D^{2} u\right)+\lambda u+|D u|^{p} \leq f(x),
$$

where $\lambda \geq 0, x \mapsto A(x)$ is a continuous map from $\Omega$ into the space $\mathcal{S}_{N}^{+}$of nonnegative symmetric matrices of order $N$, and $f$ is a continuous function. In all of this section, the exponent $p$ will be assumed to satisfy $p>2$. 
2.1. Local Hölder estimates. Let us start by considering viscosity solutions of inequality (2.1) posed in a ball of $\mathbb{R}^{N}$. Here and in the following, we use the notation $B_{r}\left(x_{0}\right)$ for the open ball centered at $x_{0} \in \mathbb{R}^{N}$ and having radius $r>0$.

Lemma 2.1. Let $B$ be any open ball in $\mathbb{R}^{N}$ and let $d_{\partial B}$ denote the distance function from the boundary of $B$. If $u \in U S C(B)$ is a bounded viscosity subsolution of equation (2.1) in $B$ with $p>2$, then

$$
u(x)-u(y) \leq K\left(\frac{|x-y|}{d_{\partial B}(x)^{1-\alpha}}+|x-y|^{\alpha}\right) \quad \forall x, y \in B,
$$

where $\alpha=\frac{p-2}{p-1}$ and $K$ is a positive constant depending only on $p,\|A\|_{L^{\infty}(B)}$ and $\left\|d_{\partial B}^{p(1-\alpha)}\left(f^{+}+\lambda u^{-}\right)\right\|_{L^{\infty}(B)}$.

Proof. Let $B=B_{r}\left(x_{0}\right)$ and let us select a smooth monotone radial function $d \in$ $C^{2}(\bar{B})$ satisfying the following properties:

$$
\left\{\begin{array}{l}
d(x)=d_{\partial B}(x)=r-\left|x-x_{0}\right| \quad \text { if } \frac{r}{2} \leq\left|x-x_{0}\right| \leq r, \\
c_{0} d_{\partial B}(x) \leq d(x) \leq d_{\partial B}(x) \quad \forall x \in \bar{B}, \\
|D d(x)| \leq 1, \quad-\frac{c_{1}}{r} I_{N} \leq D^{2} d(x) \leq O_{N} \quad \forall x \in \bar{B},
\end{array}\right.
$$

for some positive constants $c_{0}$ and $c_{1}$, and with $I_{N}$ and $O_{N}$ standing for, respectively, the unit and the null squared matrix of order $N$.

We consider, for fixed $y \in B$ and $k, L>0$ to be specified later, the function

$$
w_{y, k, L}(x)=u(x)-u(y)-k\left(\frac{|x-y|}{d(x)^{1-\alpha}}+L|x-y|^{\alpha}\right),
$$

and we set

$$
M_{y, k, L}=\sup _{x \in B} w_{y, k, L}(x) .
$$

We claim that for $k$ and $L$ large enough, one has $M_{y, k, L} \leq 0$. For, assume by contradiction that

$$
M_{y, k, L}>0 .
$$

In this case, $M_{y, k, L}$ is in fact a maximum achieved inside $B \backslash\{y\}$, by the upper semicontinuity of $u$ and since

$$
w_{y, k, L}(y)=0 \quad \text { and } \quad \lim _{x \rightarrow \partial B} w_{y, k, L}(x)=-\infty .
$$

Then, there exists $\hat{x} \in B$ (let us omit the dependence on $y, k, L$ for convenience), $\hat{x} \neq y$, such that $u-\Phi$ has a maximum at $\hat{x}$, where

$$
\Phi(x)=u(y)+k\left(\frac{|x-y|}{d(x)^{1-\alpha}}+L|x-y|^{\alpha}\right) .
$$

Since $u$ is a viscosity subsolution of (2.1) and $\Phi(x)$ is a smooth function around $\hat{x}$, then we have

$$
-\operatorname{tr}\left(A(\hat{x}) D^{2} \Phi(\hat{x})\right)+|D \Phi(\hat{x})|^{p} \leq f(\hat{x})-\lambda u(\hat{x}) \leq f^{+}(\hat{x})+\lambda u^{-}(\hat{x}),
$$

with $f^{+}=f \vee 0$ and $u^{-}=-u \wedge 0$. Computing we get

$$
D \Phi(x)=k\left[\left(d(x)^{\alpha-1}+\alpha L|x-y|^{\alpha-1}\right) \frac{x-y}{|x-y|}-(1-\alpha) d(x)^{\alpha-2}|x-y| D d(x)\right]
$$


and

$$
\begin{aligned}
D^{2} \Phi(x)=k & {\left[\frac{\left(d(x)^{\alpha-1}+\alpha L|x-y|^{\alpha-1}\right)}{|x-y|}\left(I_{N}-\frac{x-y}{|x-y|} \otimes \frac{x-y}{|x-y|}\right)\right.} \\
& -(1-\alpha) d(x)^{\alpha-2}\left(\frac{x-y}{|x-y|} \otimes D d(x)+D d(x) \otimes \frac{x-y}{|x-y|}\right) \\
& +(1-\alpha)(2-\alpha)|x-y| d(x)^{\alpha-3} \operatorname{Dd}(x) \otimes D d(x) \\
& -(1-\alpha)|x-y| d(x)^{\alpha-2} D^{2} d(x) \\
& \left.-\alpha(1-\alpha) L|x-y|^{\alpha-2} \frac{x-y}{|x-y|} \otimes \frac{x-y}{|x-y|}\right] .
\end{aligned}
$$

We then have, setting $\eta=\frac{x-y}{|x-y|}$,

$$
\begin{aligned}
\operatorname{tr}\left(A(x) D^{2} \Phi(x)\right)=k[ & \left(\frac{d(x)^{\alpha-1}}{|x-y|}+\alpha L|x-y|^{\alpha-2}\right)(\operatorname{tr}(A(x))-A(x) \eta \cdot \eta) \\
& -2(1-\alpha) d(x)^{\alpha-2} A(x) \eta \cdot D d(x) \\
& +(1-\alpha)(2-\alpha)|x-y| d(x)^{\alpha-3} A(x) D d(x) \cdot D d(x) \\
& -(1-\alpha)|x-y| d(x)^{\alpha-2} \operatorname{tr}\left(A(x) D^{2} d(x)\right) \\
& \left.-\alpha(1-\alpha) L|x-y|^{\alpha-2} A(x) \eta \cdot \eta\right] .
\end{aligned}
$$

By (2.3) and since $A(x)$ is defined to be nonnegative, we deduce that

$$
\operatorname{tr}\left(A(x) D^{2} d(x)\right) \geq-\frac{c_{1}}{r} \operatorname{tr}(A(x)) \geq-\frac{c_{1}}{r}\|A\|_{L^{\infty}(B)},
$$

so that, for $x, y \in B$,

$$
-|x-y| \operatorname{tr}\left(A(x) D^{2} d(x)\right) \leq 2 c_{1}\|A\|_{L^{\infty}(B)} .
$$

Therefore, by recalling also that $\alpha<1$, we obtain

$$
\begin{aligned}
\operatorname{tr}\left(A(x) D^{2} \Phi(x)\right) \leq c k\|A\|_{L^{\infty}(B)}\left(\frac{d(x)^{\alpha-1}}{|x-y|}\right. & +d(x)^{\alpha-2} \\
& \left.+|x-y| d(x)^{\alpha-3}+L|x-y|^{\alpha-2}\right),
\end{aligned}
$$

for some constant $c>0$. Henceforth we set $\xi:=\frac{|\hat{x}-y|}{d(\hat{x})}$ and we denote by the same letter $c$ several universal constants which may vary from line to line. The above inequality together with (2.5) and (2.6) then yields

$$
\begin{gathered}
k^{p} d(\hat{x})^{(\alpha-1) p}\left|\left(1+\alpha L \xi^{\alpha-1}\right) \frac{\hat{x}-y}{|\hat{x}-y|}-(1-\alpha) \xi D d(\hat{x})\right|^{p} \\
\leq f^{+}(\hat{x})+\lambda u^{-}(\hat{x})+c k\|A\|_{L^{\infty}(B)} d(\hat{x})^{\alpha-2}\left(1+\xi+\frac{1}{\xi}+L \xi^{\alpha-2}\right) ;
\end{gathered}
$$


hence

$$
\begin{aligned}
& k^{p}\left|\left(1+\alpha L \xi^{\alpha-1}\right) \frac{\hat{x}-y}{|\hat{x}-y|}-(1-\alpha) \xi D d(\hat{x})\right|^{p} \\
& \leq d(\hat{x})^{p(1-\alpha)}\left(f^{+}(\hat{x})+\lambda u^{-}(\hat{x})\right) \\
& \quad+c k\|A\|_{L^{\infty}(B)} d(\hat{x})^{\alpha-2-p(\alpha-1)}\left(1+\xi+\frac{1}{\xi}+L \xi^{\alpha-2}\right) .
\end{aligned}
$$

Now observe that, since the function $d(x)$ is nonnegative and concave in $B$, we have

$$
0 \leq d(y) \leq d(x)-D d(x) \cdot(x-y) \quad \forall x, y \in B,
$$

which implies

$$
\operatorname{Dd}(x) \cdot \frac{x-y}{|x-y|} \leq \frac{d(x)}{|x-y|} \quad \forall x, y \in B .
$$

This yields the estimate

$$
\begin{gathered}
\left|\left(1+\alpha L \xi^{\alpha-1}\right) \frac{\hat{x}-y}{|\hat{x}-y|}-(1-\alpha) \xi D d(\hat{x})\right|^{2} \\
\geq\left(1+\alpha L \xi^{\alpha-1}\right)^{2}+(1-\alpha)^{2} \xi^{2}|D d(\hat{x})|^{2}-2(1-\alpha)\left(1+\alpha L \xi^{\alpha-1}\right) .
\end{gathered}
$$

From Young's inequality we then obtain

$$
\begin{gathered}
\left|\left(1+\alpha L \xi^{\alpha-1}\right) \frac{\hat{x}-y}{|\hat{x}-y|}-(1-\alpha) \xi D d(\hat{x})\right|^{2} \\
\geq \frac{1}{2}\left(1+\alpha L \xi^{\alpha-1}\right)^{2}+(1-\alpha)^{2} \xi^{2}|D d(\hat{x})|^{2}-2(1-\alpha)^{2} .
\end{gathered}
$$

Moreover, by (2.3) we have $|D d(\hat{x})|=1$ if $d(\hat{x}) \leq r / 2$ and $\xi=\frac{|\hat{x}-y|}{d(\hat{x})} \leq 4$ if $d(\hat{x}) \geq r / 2$. Hence

$$
\left|\left(1+\alpha L \xi^{\alpha-1}\right) \frac{\hat{x}-y}{|\hat{x}-y|}-(1-\alpha) \xi D d(\hat{x})\right|^{2} \geq \frac{1}{2}\left(1+\alpha L \xi^{\alpha-1}\right)^{2}+(1-\alpha)^{2} \xi^{2}-c .
$$

Next, we choose $L>1$ sufficiently large depending on $\alpha$ such that

$$
\frac{1}{2}\left(1+\alpha L \xi^{\alpha-1}\right)^{2}+(1-\alpha)^{2} \xi^{2}-c \geq \frac{1}{4}\left[\left(1+\alpha L \xi^{\alpha-1}\right)^{2}+(1-\alpha)^{2} \xi^{2}\right] \quad \forall \xi>0,
$$

so that

$$
\left|\left(1+\alpha L \xi^{\alpha-1}\right) \frac{\hat{x}-y}{|\hat{x}-y|}-(1-\alpha) \xi D d(\hat{x})\right|^{2} \geq \frac{1}{4}\left[\left(1+\alpha L \xi^{\alpha-1}\right)^{2}+(1-\alpha)^{2} \xi^{2}\right] .
$$

By using (2.8), together with (2.7), we obtain, since $p>2$,

$$
\begin{aligned}
& \left(\frac{k}{2}\right)^{p}\left[1+\left(\alpha L \xi^{\alpha-1}\right)^{p}+((1-\alpha) \xi)^{p}\right] \\
& \leq\left\|d^{p(1-\alpha)}\left(f^{+}+\lambda u^{-}\right)\right\|_{L^{\infty}(B)} \\
& \quad+c k\|A\|_{L^{\infty}(B)} d(\hat{x})^{\alpha-2-p(\alpha-1)}\left(1+\xi+\frac{1}{\xi}+L \xi^{\alpha-2}\right) .
\end{aligned}
$$


The choice $\alpha=\frac{p-2}{p-1}$ gives $p(\alpha-1)=\alpha-2$; hence

$$
\begin{gathered}
\left(\frac{k}{2}\right)^{p}\left[1+\left(\alpha L \xi^{\alpha-1}\right)^{p}+((1-\alpha) \xi)^{p}\right] \\
\leq\left\|d^{p(1-\alpha)}\left(f^{+}+\lambda u^{-}\right)\right\|_{L^{\infty}(B)}+c k\|A\|_{L^{\infty}(B)}\left(1+\xi+\frac{1}{\xi}+L \xi^{p(\alpha-1)}\right) .
\end{gathered}
$$

Moreover, since $p(\alpha-1)=\alpha-2<-1$ and $L>1$, it easily follows that

$$
\begin{gathered}
\left(\frac{\alpha \wedge(1-\alpha)}{2}\right)^{p} k^{p}\left(1+L^{p} \xi^{p(\alpha-1)}+\xi^{p}\right) \\
\leq\left\|d^{p(1-\alpha)}\left(f^{+}+\lambda u^{-}\right)\right\|_{L^{\infty}(B)}+c k\|A\|_{L^{\infty}(B)}\left(1+L^{p} \xi^{p(\alpha-1)}+\xi^{p}\right) .
\end{gathered}
$$

We conclude that, if $k \gg\|A\|_{L^{\infty}(B)}^{1 /(p-1)}$, then (2.4) implies that

$$
k \leq c\left\|d^{p(1-\alpha)}\left(f^{+}+\lambda u^{-}\right)\right\|_{L^{\infty}(B)}^{1 / p} .
$$

Therefore, for

$$
k \gg\|A\|_{L^{\infty}(B)}^{1 /(p-1)} \vee\left\|d^{p(1-\alpha)}\left(f^{+}+\lambda u^{-}\right)\right\|_{L^{\infty}(B)}^{1 / p}
$$

we obtain a contradiction showing that (2.4) cannot hold. This means that

$$
u(x)-u(y) \leq k\left(\frac{|x-y|}{d(x)^{1-\alpha}}+L|x-y|^{\alpha}\right) \quad \forall x, y \in B,
$$

so that, by also recalling (2.3), inequality (2.2) is proved to hold with

$$
K=c\left(\|A\|_{L^{\infty}(B)}^{1 /(p-1)} \vee\left\|d_{\partial B}^{p(1-\alpha)}\left(f^{+}+\lambda u^{-}\right)\right\|_{L^{\infty}(B)}^{1 / p}\right) .
$$

Remark 2.2. In the completely degenerate case $A(x) \equiv O_{N}$, inequality (2.9) implies

$$
k^{p} \leq 2^{p}\left\|d_{\partial B}^{p(1-\alpha)}\left(f^{+}+\lambda u^{-}\right)\right\|_{L^{\infty}(B)}
$$

for any $0<\alpha<1$. This yields that estimate (2.2) holds for any $\alpha<1$ with a constant $K>0$ depending only on $p$ and $\left\|d_{\partial B}^{p(1-\alpha)}\left(f^{+}+\lambda u^{-}\right)\right\|_{L^{\infty}(B)}$. By letting $\alpha \rightarrow 1$ one recovers the Lipschitz regularity which is known to hold for subsolutions of coercive first order equations (see e.g. [1, 2, 12]).

We observe that, by reversing the roles of $x$ and $y$ in inequality (2.2), under the assumptions of Lemma 2.1 any subsolution $u$ satisfies

$$
|u(x)-u(y)| \leq K\left[\frac{|x-y|}{\left(d_{\partial B}(x) \wedge d_{\partial B}(y)\right)^{1-\alpha}}+|x-y|^{\alpha}\right] \quad \forall x, y \in B .
$$

This immediately implies that $u$ is actually continuous in $B$. More than that, we have the following result.

Lemma 2.3. Let $u: B \rightarrow \mathbb{R}$ be a function satisfying inequality (2.10) for some $0<\alpha<1$. Then $u$ can be extended up to the boundary as a function in $C^{0, \alpha}(\bar{B})$, 
and

$$
|u(x)-u(y)| \leq K_{1}|x-y|^{\alpha} \quad \forall x, y \in \bar{B},
$$

with a constant $K_{1}>0$ depending only on $K$ and $\alpha$.

Proof. First we consider the one-dimensional case: assume that $u:(0,1) \rightarrow \mathbb{R}$ satisfies

$$
|u(s)-u(t)| \leq K\left[\frac{|s-t|}{(s \wedge t)^{1-\alpha}}+|s-t|^{\alpha}\right] \quad \forall s, t \in(0,1) .
$$

For example, let $s<t$, and consider the sequence of points $a_{k}=s+\frac{t-s}{2^{k}}$. Then $a_{k}$ is a decreasing sequence starting from $a_{0}=t$ and converging to $s$. By (2.12) we have

$$
\left|u\left(a_{n}\right)-u(t)\right| \leq \sum_{k=1}^{n}\left|u\left(a_{k}\right)-u\left(a_{k-1}\right)\right| \leq K \sum_{k=1}^{n}\left[\frac{\left(a_{k-1}-a_{k}\right)}{a_{k}^{1-\alpha}}+\left(a_{k-1}-a_{k}\right)^{\alpha}\right] .
$$

Since $a_{k-1}-a_{k}=2\left(a_{k}-a_{k+1}\right)=\frac{(t-s)}{2^{k}}$, we get

$$
\begin{aligned}
\left|u\left(a_{n}\right)-u(t)\right| & \leq K \sum_{k=1}^{n}\left[2 \frac{\left(a_{k}-a_{k+1}\right)}{a_{k}^{1-\alpha}}+\frac{(t-s)^{\alpha}}{2^{\alpha k}}\right] \\
& \leq K\left[\sum_{k=1}^{n} 2 \int_{a_{k+1}}^{a_{k}} r^{\alpha-1} d r+\frac{(t-s)^{\alpha}}{2^{\alpha}-1}\right] \\
& \leq K\left[2 \int_{s}^{t} r^{\alpha-1} d r+\frac{(t-s)^{\alpha}}{2^{\alpha}-1}\right] \\
& \leq K\left(\frac{2}{\alpha}+\frac{1}{2^{\alpha}-1}\right)|t-s|^{\alpha}=K^{\prime}|t-s|^{\alpha} .
\end{aligned}
$$

Letting $n \rightarrow \infty$ gives the conclusion. This shows that a function $u: B \rightarrow \mathbb{R}$ satisfying (2.10) is $\alpha$-Hölder continuous when restricted along any radius of the ball $B$.

In order to extend inequality (2.11) to all $x, y \in B$, we use the same argument as in [13. Suppose for example that $d_{\partial B}(x) \leq d_{\partial B}(y)$ and write $x=\bar{x}+d_{\partial B}(x) \nu(\bar{x})$, $y=\bar{y}+d_{\partial B}(y) \nu(\bar{y})$, where $\bar{x}, \bar{y}$ are the projections of $x, y$ on $\partial B$ and $\nu$ is the inward normal vector field to $\partial B$. We distinguish several cases: if $|x-y| \leq d_{\partial B}(y)$, then consider the point $z=\bar{x}+d_{\partial B}(y) \nu(\bar{x})$, which has the same distance of $y$ but lies on the same radius as $x$. From the one dimensional result and (2.10) we obtain

$$
\begin{aligned}
|u(x)-u(y)| & \leq|u(x)-u(z)|+|u(z)-u(y)| \\
& \leq K^{\prime}\left[\left|d_{\partial B}(x)-d_{\partial B}(y)\right|^{\alpha}+\frac{|z-y|}{d_{\partial B}(y)^{1-\alpha}}+|z-y|^{\alpha}\right]
\end{aligned}
$$

and since $|z-y| \leq|x-y|+\left|d_{\partial B}(x)-d_{\partial B}(y)\right| \leq 2|x-y|$ and $|x-y| \leq d_{\partial B}(y)$, we deduce (2.11).

On the other hand, if $r>|x-y|>d_{\partial B}(y)$, then we consider the points $w_{1}=$ $\bar{x}+|x-y| \nu(\bar{x})$ and $w_{2}=\bar{y}+|x-y| \nu(\bar{y})$, which are, respectively, on the $\bar{x}$-normal and on the $\bar{y}$-normal at same distance $|x-y|$ from the boundary. Similarly as 
before we have

$$
\begin{aligned}
|u(x)-u(y)| \leq & \left|u(x)-u\left(w_{1}\right)\right|+\left|u\left(w_{2}\right)-u(y)\right|+\left|u\left(w_{1}\right)-u\left(w_{2}\right)\right| \\
\leq & K^{\prime}\left[|| x-y\left|-d_{\partial B}(x)\right|^{\alpha}+|| x-y\left|-d_{\partial B}(y)\right|^{\alpha}\right. \\
& \left.\quad+\frac{\left|w_{1}-w_{2}\right|}{|x-y|^{1-\alpha}}+\left|w_{1}-w_{2}\right|^{\alpha}\right]
\end{aligned}
$$

and since we are in the case $|x-y|>d_{\partial B}(y) \geq d_{\partial B}(x)$ we conclude again (2.11).

Finally, if $|x-y| \geq r$, we consider the center $x_{0}$ of $B$ and we obtain

$$
|u(x)-u(y)| \leq\left|u(x)-u\left(x_{0}\right)\right|+\left|u(y)-u\left(x_{0}\right)\right| \leq 2 K^{\prime} r^{\alpha} \leq 2 K^{\prime}|x-y|^{\alpha} .
$$

Lemma 2.1 and Lemma 2.3 jointly yield the following local Hölder estimate.

Theorem 2.4. Let $\Omega$ be an open bounded subset of $\mathbb{R}^{N}$, and let $u \in U S C(\Omega)$ be a bounded viscosity solution of

$$
-\operatorname{tr}\left(A(x) D^{2} u\right)+\lambda u+|D u|^{p} \leq f(x), \quad x \in \Omega
$$

with $\lambda \geq 0, p>2, A: \Omega \rightarrow \mathcal{S}_{N}^{+}$bounded and continuous and $f: \Omega \rightarrow \mathbb{R}$ continuous such that $d_{\partial \Omega}^{p / p-1} f^{+}$is bounded, $d_{\partial \Omega}$ being the distance function from $\partial \Omega$.

Then, $u$ is locally Hölder continuous in $\Omega$, and there exists a positive constant $K_{1}$ depending only on $p,\|A\|_{L^{\infty}(\Omega)}$ and $\left\|d_{\partial \Omega}^{p / p-1}\left(f^{+}+\lambda u^{-}\right)\right\|_{L^{\infty}(\Omega)}$ such that

$$
|u(x)-u(y)| \leq K_{1}|x-y|^{\alpha} \quad \forall x, y \in B,
$$

for any open ball $B \subset \Omega$ and with $\alpha=\frac{p-2}{p-1}$.

Remark 2.5. In Theorem 2.4 the exponent $\alpha=\frac{p-2}{p-1}$ is the best one can expect. Indeed, a direct computation shows that the function $u(x)=|x|^{(p-2) /(p-1)}$ satisfies

$$
-\Delta u+\lambda u+|D u|^{p} \leq \lambda \quad \text { in } B_{1}(0) \subset \mathbb{R}^{N},
$$

as soon as $N \geq 2$.

2.2. Global Hölder estimates. This section is devoted to extending Theorem 2.4. in order to prove the global Hölder continuity of any subsolution $u$ without imposing any boundary condition on $u$. Once $u$ is known to be uniformly locally Hölder continuous in a domain $\Omega \subset \mathbb{R}^{N}$ (as stated in Theorem 2.4), its global Hölder continuity can be obtained under some smoothness assumptions on the boundary of $\Omega$.

Henceforth, we say that an open bounded subset $\Omega \subset \mathbb{R}^{N}$ has Lipschitz boundary if around each point $x_{0} \in \partial \Omega$ the domain $\Omega$ can be represented as the subgraph of a Lipschitz continuous function of $N-1$ variables.

We also say that $\Omega$ satisfies the uniform interior sphere condition, with a radius $r>0$, if for every $x_{0} \in \partial \Omega$ there exists a unit vector $\nu\left(x_{0}\right)$ such that

$$
B_{r}\left(x_{0}+r \nu\left(x_{0}\right)\right) \subset \Omega .
$$

Note that any such unit vector $\nu\left(x_{0}\right)$ belongs to the normal cone to $\mathbb{R}^{n} \backslash \Omega$ at $x_{0}$ (see e.g. 7] for the definiton of tangent and normal cones to closed sets).

Lemma 2.6. Let $\Omega \subset \mathbb{R}^{N}$ be an open bounded domain having Lipschitz boundary and satisfying the uniform interior sphere condition. If $u: \Omega \rightarrow \mathbb{R}$ is a continuous function such that there exist $K_{1}>0$ and $0<\alpha<1$ satisfying

$$
|u(x)-u(y)| \leq K_{1}|x-y|^{\alpha} \quad \forall x, y \in B,
$$


for any open ball $B \subset \Omega$, then $u$ can be extended up to $\partial \Omega$ as a function satisfying

$$
|u(x)-u(y)| \leq M|x-y|^{\alpha}, \quad \forall x, y \in \bar{\Omega},
$$

where $M \geq K_{1}$ is a constant depending on $\alpha, K_{1}$ and $\partial \Omega$.

Proof. By the interior sphere property, every point $x \in \partial \Omega$ also lies on the boundary of some open ball contained in $\Omega$. In particular, for every $x \in \partial \Omega$, let $B^{x} \subset \Omega$ be the largest open ball such that $x \in \partial B^{x}$. Thanks to assumption (2.13), we can therefore define the function $u$ at $x \in \partial \Omega$ by setting

$$
u(x)=\lim _{\substack{y \rightarrow x \\ y \in B^{x}}} u(y) .
$$

We note that with this definition, for every $x \in \Omega$ and for any $\bar{x} \in \partial \Omega$ such that $|x-\bar{x}|=d_{\partial \Omega}(x),(2.13)$ implies that

$$
|u(z)-u(\bar{x})| \leq K_{1}|z-\bar{x}|^{\alpha}, \quad \forall z \in B_{d_{\partial \Omega}(x)}(x) .
$$

Let $x, y \in \bar{\Omega}$ be distinct points and let us prove (2.14).

If $|x-y|<d_{\partial \Omega}(x) \vee d_{\partial \Omega}(y)$, then both $x$ and $y$ lie in $\Omega$ and either $y \in B_{d_{\partial \Omega}(x)}(x)$ or $x \in B_{d_{\partial \Omega}(y)}(y)$. In both cases, (2.14) follows directly from (2.15) with $M=K_{1}$.

Hence, we can assume that $|x-y| \geq d_{\partial \Omega}(x) \vee d_{\partial \Omega}(y)$. If $\bar{x}, \bar{y} \in \partial \Omega$ are boundary points such that $|x-\bar{x}|=d_{\partial \Omega}(x)$ and $|y-\bar{y}|=d_{\partial \Omega}(y)$, then by (2.15) we have

$$
\begin{aligned}
|u(x)-u(y)| & \leq|u(x)-u(\bar{x})|+|u(y)-u(\bar{y})|+|u(\bar{x})-u(\bar{y})| \\
& \leq K_{1}\left(d_{\partial \Omega}(x)^{\alpha}+d_{\partial \Omega}(y)^{\alpha}\right)+|u(\bar{x})-u(\bar{y})| \\
& \leq 2 K_{1}|x-y|^{\alpha}+|u(\bar{x})-u(\bar{y})| .
\end{aligned}
$$

If we show that

$$
|u(x)-u(y)| \leq K_{2}|x-y|^{\alpha}, \quad \forall x, y \in \partial \Omega,
$$

then from the above we obtain

$$
|u(x)-u(y)| \leq 2 K_{1}|x-y|^{\alpha}+K_{2}|\bar{x}-\bar{y}|^{\alpha} \leq\left(2 K_{1}+3^{\alpha} K_{2}\right)|x-y|^{\alpha},
$$

that is, (2.14). Therefore, it is enough to prove (2.16).

We claim that, by the Lipschitz regularity of $\partial \Omega$, there exists $\delta>0$ such that for all $x, y \in \partial \Omega$ one has

$$
|x-y|<\delta \Longrightarrow|\nu(x)-\nu(y)|<2-\delta,
$$

for any choice of unit vectors $\nu(x)$ and $\nu(y)$ belonging to the normal cones at $x$ and $y$, respectively. Indeed, if not, we could find sequences $\left\{x_{k}\right\}$ and $\left\{y_{k}\right\}$ of boundary points converging to the same point $x_{0} \in \partial \Omega$ and such that, for every $k$, there exist normal unit vectors $\nu\left(x_{k}\right)$ and $\nu\left(y_{k}\right)$ satisfying $\left|\nu\left(x_{k}\right)-\nu\left(y_{k}\right)\right| \geq 2-1 / k$. By letting $k \rightarrow \infty$, we would obtain two unit vectors $\nu_{1}$ and $\nu_{2}$ both in the normal cone at $x_{0}$ satisfying $\left|\nu_{1}-\nu_{2}\right|=2$, that is, such that $\nu_{1}=-\nu_{2}$. But this is impossible for a Lipschitz domain (see Corollary 2 and Theorem 3 in [15).

We set $\delta_{0}=\delta \wedge \delta r$, where $r>0$ is such that $\Omega$ satisfies the uniform interior sphere condition with radius $r$, and let $x, y \in \partial \Omega$ with $|x-y|<\delta_{0}$. By the uniform interior sphere condition, there exist unit vectors $\nu(x)$ and $\nu(y)$ such that

$$
B_{\frac{|x-y|}{\delta}}\left(x+\frac{|x-y|}{\delta} \nu(x)\right), B_{\frac{|x-y|}{\delta}}\left(y+\frac{|x-y|}{\delta} \nu(y)\right) \subset \Omega .
$$


Moreover, by (2.17) one has

$$
\begin{aligned}
\left|\left(x+\frac{|x-y|}{\delta} \nu(x)\right)-\left(y+\frac{|x-y|}{\delta} \nu(y)\right)\right| & \leq|x-y|+\frac{|x-y|}{\delta}|\nu(x)-\nu(y)| \\
& <2 \frac{|x-y|}{\delta},
\end{aligned}
$$

which implies that there exists a point

$$
z \in B_{\frac{|x-y|}{\delta}}\left(x+\frac{|x-y|}{\delta} \nu(x)\right) \cap B_{\frac{|x-y|}{\delta}}\left(y+\frac{|x-y|}{\delta} \nu(y)\right) .
$$

By (2.15), it then follows that

$$
\begin{aligned}
|u(x)-u(y)| & \leq|u(x)-u(z)|+|u(y)-u(z)| \\
& \leq K_{1}\left(|x-z|^{\alpha}+|y-z|^{\alpha}\right) \leq \frac{2^{\alpha+1}}{\delta^{\alpha}} K_{1}|x-y|^{\alpha} .
\end{aligned}
$$

Inequality (2.16) is thus proved for $x, y \in \partial \Omega$ satisfying $|x-y|<\delta_{0}$. By covering $\partial \Omega$ with a finite number $n$ of balls of radius $\delta_{0}$, we get (2.16) with a constant $K_{2}$ depending on $K_{1}, \alpha, \delta, r$ and the number $n$.

As a consequence of Theorem 2.4 and Lemma 2.6 we can state the following global Hölder estimate.

Theorem 2.7. Let $\Omega \subset \mathbb{R}^{N}$ be an open bounded domain having Lipschitz boundary and satisfying the uniform interior sphere condition. Let $u \in U S C(\Omega)$ be a bounded viscosity solution of

$$
-\operatorname{tr}\left(A(x) D^{2} u\right)+\lambda u+|D u|^{p} \leq f(x), \quad x \in \Omega,
$$

with $\lambda \geq 0, p>2, A: \Omega \rightarrow \mathcal{S}_{N}^{+}$bounded and continuous and $f: \Omega \rightarrow \mathbb{R}$ continuous such that $d_{\partial \Omega}^{p / p-1} f^{+}$is bounded, $d_{\partial \Omega}$ being the distance function from $\partial \Omega$.

Then, $u$ is Hölder continuous in $\bar{\Omega}$, and there exists a positive constant $M$ depending only on $p,\|A\|_{L^{\infty}(\Omega)},\left\|d_{\partial \Omega}^{p / p-1}\left(f^{+}+\lambda u^{-}\right)\right\|_{L^{\infty}(\Omega)}$ and on $\partial \Omega$ such that

$$
|u(x)-u(y)| \leq M|x-y|^{\alpha} \quad \forall x, y \in \bar{\Omega}, \alpha=\frac{p-2}{p-1} .
$$

Remark 2.8. According to Remark [2.2, if $A(x) \equiv 0$ in $\Omega$, then any bounded subsolution $u$ is such that $|D u| \in L^{\infty}(\Omega)$. In this case, in order to obtain that $u$ is Lipschitz continuous in $\bar{\Omega}$, it is enough to assume that $\Omega$ has Lipschitz boundary.

Remark 2.9. If $\Omega$ is a convex domain of class $C^{2}$, we can avoid passing through the local estimate of Theorem 2.4 in order to get the global regularity. In this case, we can apply the arguments of the proof of Lemma 2.1 by replacing $B$ with $\Omega$, and then, thanks to Lemma 2.3, we obtain directly the global estimate (2.18). Actually, even if $\Omega$ is not convex, but it is of class $C^{2}$, the proof of Lemma 2.1 can be slightly modified so as to yield (jointly with Lemma 2.3) estimate (2.18) with a constant $M$ depending not only on $\left\|\lambda u^{-}\right\|_{L^{\infty}(\Omega)}$ but also on $\|u\|_{L^{\infty}(\Omega)}$. Thus, the use of the local estimate given by Theorem 2.4 is needed both for relaxing the assumptions on the smoothness of $\partial \Omega$ and for having estimate (2.18) with a constant $M$ independent of $\|u\|_{L^{\infty}(\Omega)}$. 
Remark 2.10. The continuity assumption on $f(x)$ and $A(x)$ plays no role in the estimate of Lemma 2.1 and in the result of Theorem 2.7. It was assumed only in order to consider the standard (pointwise) definition of viscosity subsolutions. The same results hold if $f(x), A(x)$ are assumed to be measurable and essentially bounded, and considering subsolutions $u$ in the so-called $L^{p}$-viscosity sense introduced in [6].

It is clear from the proof of Lemma 2.1 that all the results of this section hold in more generality and in particular for some fully nonlinear operators as well.

More precisely, the same conclusions of Theorem 2.4 and Theorem 2.7 hold if $u \in U S C(\Omega)$ is a bounded viscosity solution of

$$
F\left(x, D u, D^{2} u\right)+\lambda u+|D u|^{p} \leq f(x), \quad x \in \Omega,
$$

where $\lambda, p, f(x)$ satisfy the assumptions of the above theorems and $F(x, \xi, M)$ is a continuous function, degenerate elliptic, i.e.

$$
F\left(x, \xi, M_{1}\right)-F\left(x, \xi, M_{2}\right) \leq 0 \quad \forall x \in \Omega, \xi \in \mathbb{R}^{N}, M_{1}, M_{2} \in \mathcal{S}_{N}: M_{1} \geq M_{2}
$$

and satisfying, for some positive constants $\Lambda, \gamma>0$ :

$$
F(x, \xi, M) \geq-\Lambda\|M\| \quad \forall x \in \Omega, \xi \in \mathbb{R}^{N}:|\xi|>\gamma, M \in \mathcal{S}_{N} .
$$

Moreover, the same approach can provide similar results in even more general situations, including quasilinear operators such as the $m$-Laplacian. For the sake of clarity, let us state precisely this extended result.

Theorem 2.11. Let $\Omega \subset \mathbb{R}^{N}$ be an open bounded domain having Lipschitz boundary and satisfying the uniform interior sphere condition. Let $F(x, \xi, M)$ be a continuous function satisfying (2.20) and the growth assumption

$$
F(x, \xi, M) \geq-\Lambda|\xi|^{m-2}\|M\| \quad \forall x \in \Omega, \xi \in \mathbb{R}^{N}:|\xi|>\gamma, M \in \mathcal{S}_{N}
$$

for some constants $\Lambda, \gamma>0$ and for some $m>1$, and let $u \in U S C(\Omega)$ be a bounded viscosity solution of (2.19), where $\lambda \geq 0, p>m, f: \Omega \rightarrow \mathbb{R}$ is continuous and such that $d_{\partial \Omega}^{p / p-(m-1)} f^{+}$is bounded.

Then, $u$ is Hölder continuous in $\bar{\Omega}$, and there exists a positive constant $M$ depending on $p, m, \Lambda, \gamma,\left\|d_{\partial \Omega}^{p / p-(m-1)}\left(f^{+}+\lambda u^{-}\right)\right\|_{L^{\infty}(\Omega)}$ and on $\partial \Omega$ such that

$$
|u(x)-u(y)| \leq M|x-y|^{\alpha} \quad \forall x, y \in \bar{\Omega}, \alpha=\frac{p-m}{p-(m-1)} .
$$

The proof of this result follows exactly the same steps as for Theorem 2.7 the only minor change appearing in the basic estimate of Lemma 2.1. Here it is enough to observe that, by construction of the test function $\Phi$, we have (see (2.6) and (2.8)

$$
\begin{aligned}
\frac{1}{4}\left[\left(1+\alpha L \xi^{\alpha-1}\right)+(1-\alpha) \xi\right] k d(x)^{\alpha-1} & \leq|D \Phi| \\
& \leq k d(x)^{\alpha-1}\left[\left(1+\alpha L \xi^{\alpha-1}\right)+(1-\alpha) \xi\right],
\end{aligned}
$$

which allows us to take care of the weight $|D \Phi|^{m-2}$ with similar estimates from above and from below. 
2.3. Solvability of the Dirichlet problem. In this section we address the problem of existence of solutions of (1.1) complemented with Dirichlet type boundary conditions, namely

$$
\left\{\begin{aligned}
-\operatorname{tr}\left(A(x) D^{2} u\right)+\lambda u+|D u|^{p} & =f(x) \quad \text { in } \Omega, \\
u & =\varphi \quad \text { on } \partial \Omega .
\end{aligned}\right.
$$

We assume the following standard conditions:

$$
\begin{gathered}
\Omega \subset \mathbb{R}^{N} \text { open, bounded, of class } C^{2}, \text { with } N \geq 2, \\
f \in C(\bar{\Omega}), \\
\lambda>0, \\
\varphi \in C(\partial \Omega), \\
\exists \text { a Lipschitz map } \Sigma: \bar{\Omega} \rightarrow \mathcal{M}_{N \times M} \text { : such that } \\
A(x)=\Sigma(x) \Sigma(x)^{T},
\end{gathered}
$$

where $\mathcal{M}_{N \times M}$ is the space of $N \times M$ matrices.

Moreover, we will focus on the case when the problem is nondegenerate at the boundary; more precisely, we assume that there exists a positive constant $\sigma$ such that

$$
A(x) \nu(x) \cdot \nu(x) \geq \sigma>0 \quad \forall x \in \partial \Omega .
$$

Then, it is well known that if $1<p \leq 2$, problem (2.22) has a unique strong viscosity solution, that is, a function $u \in C(\bar{\Omega})$ which is a viscosity solution in $\Omega$ and satisfies pointwisely $u=\varphi$ on $\partial \Omega$. By contrast, when $p>2$, the results of 4, 10, imply that problem (2.22), even in the uniformly elliptic case and posed in a smooth domain, cannot be solved in general. The best one can obtain is the existence of a viscosity solution satisfying the boundary condition in the relaxed viscosity formulation (see [5, 8). Precisely, in [4, the authors prove that (2.22) has a unique generalized viscosity solution, that is, a function $u \in C(\bar{\Omega})$ which is a viscosity solution in $\Omega, u \leq \varphi$ on $\partial \Omega$ and it satisfies, in the viscosity sense,

$$
x \in \partial \Omega, u(x)<\varphi(x) \Longrightarrow-\operatorname{tr}\left(A(x) D^{2} u\right)+\lambda u+|D u|^{p} \geq f(x) .
$$

In the case $p>2$, a loss of the boundary condition may actually occur. This phenomenon is consistent with the results obtained in [10] on the maximal solution of (1.1). Since, if $p>2$, the maximal solution $U$ turns out to be bounded in $\bar{\Omega}$, it is clear that every boundary datum $\varphi$ cannot be reached in the region where it exceeds $U$. In particular, the upper bound $\varphi \leq U$ is a necessary condition on $\varphi$ for the solvability of (2.22) in the strong viscosity sense.

Moreover, by Theorem 2.7, every function $u \in C(\bar{\Omega})$ which satisfies the equation in $\Omega$ is $(p-2) /(p-1)$-Hölder continuous up to the boundary. Hence, another necessary condition on $\varphi$ is the existence of $M \geq 0$ such that

$$
|\varphi(x)-\varphi(y)| \leq M|x-y|^{\alpha} \quad \forall x, y \in \partial \Omega, \alpha=\frac{p-2}{p-1} .
$$

In the next result we exhibit in the case $p>2$ some sufficient conditions ensuring the existence of a solution $u \in C(\bar{\Omega})$ of (2.22) which assumes pointwisely the boundary datum. 
Theorem 2.12. Assume that (2.23)-(2.28) hold true, and that $p>2$. There exists a constant $M_{0}>0$, depending on $p, N, \sigma, A(x)$ and on the domain $\Omega$, such that if $\varphi$ satisfies (2.29) with $M<M_{0}$ and if

$$
\lambda \inf \varphi \leq \inf f,
$$

then the Dirichlet problem (2.22) has a unique viscosity solution $u \in C^{0, \frac{p-2}{p-1}}(\bar{\Omega})$ such that $u(x)=\varphi(x)$ for every $x \in \partial \Omega$.

Proof. The uniqueness is an immediate consequence of the standard Comparison Principle for viscosity solutions (see [8]). Also the $(p-2) /(p-1)$ Hölder continuity of the solution directly follows from Theorem 2.7

Therefore, it is enough to show the existence of a solution $u \in C(\bar{\Omega})$ satisfying $u=\varphi$ on $\partial \Omega$. This is equivalent to proving the existence of a viscosity subsolution $v \in C(\bar{\Omega})$ satisfying $v=\varphi$ on $\partial \Omega$. Indeed, if such a subsolution $v$ exists, then the generalized solution $u \in C(\bar{\Omega})$ proved in 4 to exist satisfies $u \geq v$ in $\bar{\Omega}$ and $u \leq \varphi$ on $\partial \Omega$; hence $\varphi=v \leq u \leq \varphi$ on $\partial \Omega$.

We will construct the subsolution $v$ first in a small neighborhood $\mathcal{N}_{\delta}=\{x \in \Omega$ : $\left.d_{\partial \Omega}(x)<\delta\right\}$ of $\partial \Omega$, and then we will extend it to the whole $\Omega$. To this purpose, using that $\Omega$ is of class $C^{2}$, assumption (2.28) and the continuity of $A(x)$, we can fix $\delta_{0}$ such that $d_{\partial \Omega}(x)$ is a function of class $C^{2}$ in $\mathcal{N}_{\delta_{0}}$ and

$$
A(x) D d_{\partial \Omega}(x) \cdot D d_{\partial \Omega}(x) \geq \frac{\sigma}{2} \quad \forall x \in \mathcal{N}_{\delta_{0}} .
$$

For $\delta<\delta_{0}$, let us consider the function

$$
v_{y}(x)=\varphi(y)-M|x-y|^{\alpha}-\mu M d_{\partial \Omega}(x)^{\alpha},
$$

where $y \in \partial \Omega$ is fixed, $\mu>0$ will be chosen later and $\alpha=\frac{p-2}{p-1}$ as usual. Notice that $v_{y}$ is of class $C^{2}$ in $\mathcal{N}_{\delta}$, and, by a direct computation, we have

$$
\begin{aligned}
- & \operatorname{tr}\left(A(x) D^{2} v_{y}\right)+\lambda v_{y}+\left|D v_{y}\right|^{p} \\
= & \alpha M|x-y|^{\alpha-2}\left(\operatorname{tr}(A(x))+(\alpha-2) A(x) \frac{x-y}{|x-y|} \cdot \frac{x-y}{|x-y|}\right) \\
& -\mu M \alpha(1-\alpha) d_{\partial \Omega}(x)^{\alpha-2} A(x) D d_{\partial \Omega}(x) \cdot D d_{\partial \Omega}(x) \\
& +\mu M \alpha d_{\partial \Omega}(x)^{\alpha-1} \operatorname{tr}\left(A(x) D^{2} d_{\partial \Omega}(x)\right) \\
& +\lambda v_{y}(x)+M^{p} \alpha^{p}|| x-\left.y\right|^{\alpha-2}(x-y)+\left.\mu d_{\partial \Omega}(x)^{\alpha-1} D d_{\partial \Omega}(x)\right|^{p} .
\end{aligned}
$$

Since $|x-y| \geq d_{\partial \Omega}(x)$ and $(\alpha-1) p=\alpha-2$, we obtain, using (2.31),

$$
\begin{aligned}
& -\operatorname{tr}\left(A(x) D^{2} v_{y}\right)+\lambda v_{y}+\left|D v_{y}\right|^{p}-f \\
& \leq \alpha M d_{\partial \Omega}(x)^{\alpha-2}\left[N\|A\|_{L^{\infty}}-\mu\left((1-\alpha) \frac{\sigma}{2}-\delta K\|A\|_{L^{\infty}}\right)+(\alpha M)^{p-1}(1+\mu)^{p}\right] \\
& \quad+\left(\lambda v_{y}(x)-f(x)\right),
\end{aligned}
$$

for $x \in \mathcal{N}_{\delta}$ and with $K=\sup _{x \in \mathcal{N}_{\delta}}\left|D^{2} d_{\partial \Omega}(x)\right|$. By observing that

$$
v_{y} \leq \inf \varphi+M \operatorname{diam}(\Omega)^{\alpha}
$$


and by using (2.30), we deduce

$$
\begin{aligned}
& -\operatorname{tr}\left(A(x) D^{2} v_{y}\right)+\lambda v_{y}+\left|D v_{y}\right|^{p}-f \\
& \leq \alpha M d_{\partial \Omega}(x)^{\alpha-2}\left[N\|A\|_{L^{\infty}}-\mu\left((1-\alpha) \frac{\sigma}{2}-\delta K\|A\|_{L^{\infty}}\right)\right. \\
& \left.\quad+(\alpha M)^{p-1}(1+\mu)^{p}+\lambda \frac{\delta^{2-\alpha}}{\alpha} \operatorname{diam}(\Omega)^{\alpha}\right] .
\end{aligned}
$$

Hence

$$
-\operatorname{tr}\left(A(x) D^{2} v_{y}\right)+\lambda v_{y}+\left|D v_{y}\right|^{p} \leq f \quad \text { in } \mathcal{N}_{\delta}
$$

provided that

$N\|A\|_{L^{\infty}}-\mu\left((1-\alpha) \frac{\sigma}{2}-\delta K\|A\|_{L^{\infty}}\right)+(\alpha M)^{p-1}(1+\mu)^{p}+\lambda \frac{\delta^{2-\alpha}}{\alpha} \operatorname{diam}(\Omega)^{\alpha} \leq 0$.

Let us assume for the time being that (2.32) is satisfied. Then, we have proved that, for every $y \in \partial \Omega$, the function $v_{y}$ is a (classical) subsolution in $\mathcal{N}_{\delta}$. This implies that the function

$$
v_{\delta}(x)=\sup _{y \in \partial \Omega} v_{y}(x), \quad x \in \mathcal{N}_{\delta}
$$

is a subsolution as well. We notice that $v_{\delta}(x)=\varphi(x)$ for $x \in \partial \Omega$ by (2.29), and, moreover, $v_{\delta}(x) \leq \sup \varphi-M(1+\mu) \delta^{\alpha}$ for $x \in \partial \mathcal{N}_{\delta} \cap \Omega=\left\{x \in \Omega: d_{\partial \Omega}(x)=\delta\right\}$. If we further assume that

$$
\sup \varphi-\inf \varphi<M(1+\mu) \delta^{\alpha},
$$

then we can select a constant $\gamma$ such that

$$
\sup \varphi-M(1+\mu) \delta^{\alpha}<\gamma<\inf \varphi .
$$

We then define

$$
v(x)= \begin{cases}v_{\delta}(x) \vee \gamma & \text { if } x \in \mathcal{N}_{\delta}, \\ \gamma & \text { if } x \in \Omega \backslash \mathcal{N}_{\delta} .\end{cases}
$$

We notice that, by (2.30) and (2.34), $\gamma$ is a constant subsolution in $\Omega$ and, moreover, $\gamma>v_{\delta}(x)$ for every $x \in \partial \mathcal{N}_{\delta} \cap \Omega$. This implies that $v \in C(\bar{\Omega})$ is a subsolution in $\Omega$. Furthermore, again by (2.34), we have that $\gamma<v_{\delta}(x)=\varphi(x)$ for all $x \in \partial \Omega$ and then $v=\varphi$ on $\partial \Omega$.

The proof is then completed if we show that (2.32) and (2.33) hold true. From (2.29) it follows that $\sup \varphi-\inf \varphi \leq M(\operatorname{diam} \Omega)^{\alpha}$. Therefore, (2.33) is satisfied if we impose that

$$
\mu>\left(\frac{\operatorname{diam} \Omega}{\delta}\right)^{\alpha}-1
$$

Then, in order to also verify (2.32), we have to check the existence of $\mu$ and $\delta$ (with $\left.0<\delta<\delta_{0}\right)$ satisfying the above condition and such that

$$
(\alpha M)^{p-1}<\frac{\mu\left((1-\alpha) \frac{\sigma}{2}-\delta K\|A\|_{L^{\infty}(\Omega)}\right)-\left(N\|A\|_{L^{\infty}(\Omega)}+\lambda \frac{\delta^{2-\alpha}}{\alpha} \operatorname{diam}(\Omega)^{\alpha}\right)}{(1+\mu)^{p}} .
$$

Without loss of generality, we assume that $\delta$ is small enough so that $(1-\alpha) \frac{\sigma}{2}-$ $\delta K\|A\|_{L^{\infty}(\Omega)}>0$. Then, it is easy to verify the existence of $\mu$ satisfying the above 
conditions provided $M<M_{0}$, where

$M_{0}^{p-1}=\sup _{C}\left[\frac{\mu\left((1-\alpha) \frac{\sigma}{2}-\delta K_{0}\|A\|_{L^{\infty}(\Omega)}\right)-\left(N\|A\|_{L^{\infty}(\Omega)}+\lambda \frac{\delta^{2-\alpha}}{\alpha} \operatorname{diam}(\Omega)^{\alpha}\right)}{\alpha^{p-1}(1+\mu)^{p}}\right]$

with $C=\left\{(\delta, \mu): 0<\delta<\min \left(\delta_{0}, \frac{(1-\alpha) \sigma}{2 K_{0}\|A\|_{L^{\infty}(\Omega)}}\right), \mu>\left(\frac{\operatorname{diam} \Omega}{\delta}\right)^{\alpha}-1\right\}$, where $\delta_{0}$ is the value fixed above and $K_{0}=\sup _{x \in \mathcal{N}_{\delta_{0}}}\left|D^{2} d_{\partial \Omega}(x)\right|$.

Remark 2.13. Assumption (2.30) in the above theorem may be regarded as a compatibility condition among the data of the problem $\varphi, f, \lambda$. In the special case in which $\varphi(x) \equiv \varphi_{0}$ is constant, this reduces to $\lambda \varphi_{0} \leq \inf f$ and was already found in 11].

Remark 2.14. The assumption of nondegeneracy (2.28) is needed in the above result. Indeed, in the degenerate case $A(x)=0$ solutions are known to be Lipschitz continuous up to the boundary; hence $\varphi$ could not have been assumed to be just Hölder continuous. We recall that for first order equations a necessary and sufficient condition for solving the Dirichlet problem with Lipschitz boundary data can be found in [12].

\section{Lipschitz estimates: Bernstein's Method Revisited}

In this section we complement the regularity results of Section 2 by considering solutions, and not only subsolutions, of (1.1) and for the whole range $p>1$. More precisely, let $\Omega \in \mathbb{R}^{N}$ be open and bounded, and let us consider the equation

$$
-\operatorname{tr}\left(A(x) D^{2} u\right)+\lambda u+H(x, D u)=0 \quad x \in \Omega .
$$

We assume that $\lambda \geq 0$, that the map $A: \Omega \rightarrow \mathcal{S}_{N}^{+}$satisfies

$$
\begin{gathered}
\exists \text { a Lipschitz map } \Sigma: \bar{\Omega} \rightarrow \mathcal{M}_{N \times M} \text { such that } \\
A(x)=\Sigma(x) \Sigma(x)^{T},
\end{gathered}
$$

where $\mathcal{M}_{N \times M}$ is the space of $N \times M$ matrices, and that there exist positive constants $\gamma_{0}, \gamma_{1}, \gamma_{2}$ and functions $f, g_{1}, g_{2} \in C(\Omega)$ such that

$$
\begin{aligned}
& H(x, \xi) \geq \gamma_{0}|\xi|^{p}-f(x), \quad p>1, \\
& |H(x, \xi)-H(x, \eta)| \leq \gamma_{1}\left(|\xi|^{p-1}+|\eta|^{p-1}\right)|\xi-\eta|, \\
& |H(x, \xi)-H(y, \xi)| \leq \gamma_{2}\left(g_{1}(x)+g_{2}(x)|\xi|^{p}\right)|x-y| .
\end{aligned}
$$

We have the following result.

Theorem 3.1. Assume that (3.2), (3.3) hold true and that the continuous functions $f, g_{1}$ and $g_{2}$ are such that $d_{\partial \Omega}^{\frac{p}{p-1}} f, d_{\partial \Omega}^{\frac{2 p-1}{p-1}} g_{1}$ and $d_{\partial \Omega} g_{2}$ are bounded in $\Omega$, where $d_{\partial \Omega}(x)=\operatorname{dist}(x, \partial \Omega)$. Let $u \in C(\Omega)$ be a bounded viscosity solution of (3.1) in $\Omega$ with $\lambda \geq 0$.

Then $u$ is locally Lipschitz continuous and there exists a positive constant $K$ (depending on $p,\|\Sigma\|_{L^{\infty}(\Omega)},\left\|\Sigma_{x}\right\|_{L^{\infty}(\Omega)},\left\|d_{\partial \Omega}^{\frac{p}{p-1}}(f-\lambda u)^{+}\right\|_{L^{\infty}(\Omega)},\left\|d_{\partial \Omega}^{\frac{2 p-1}{p-1}} g_{1}\right\|_{L^{\infty}(\Omega)}$, $\left\|d_{\partial \Omega} g_{2}\right\|_{L^{\infty}(\Omega)}$, and $\left.\gamma_{i}, i=0,1,2\right)$ such that

$$
|D u(x)| \leq \frac{K}{d(x)^{\frac{1}{p-1}}} \quad \text { for a.e. } x \in \Omega .
$$


Proof. Let us initially assume that $\Omega=B$ is a unit ball. Then, $u \in C(B)$ is a bounded viscosity solution of equation (3.1) in $B$. We denote by $d_{\partial B}$ the distance function from the boundary of $B$, and we select a smooth monotone radial function $d \in C^{2}(\bar{B})$ satisfying the following properties:

$$
\left\{\begin{array}{l}
d(x)=d_{\partial B}(x) \quad \text { if } \quad d_{\partial B}(x) \leq \frac{1}{2}, \\
\frac{d_{\partial B}(x)}{2} \leq d(x) \leq d_{\partial B}(x) \quad \forall x \in \bar{B}, \\
|D d(x)| \leq 1, \quad D^{2} d(x) \leq 0 \quad \forall x \in \bar{B} .
\end{array}\right.
$$

Consider the function

$$
\Phi(x, y)=k|x-y| \varphi(x, y)
$$

where

$$
\varphi(x, y)=\frac{1}{d(y)^{\gamma}}\left[L+\left(\frac{|x-y|}{d(x)}\right)^{\beta}\right] .
$$

The constants $\gamma, \beta, L$ are positive and will be fixed later. Note that $\varphi(x, y)$ blows up as either $d(x)$ or $d(y)$ tend to zero; indeed $\varphi$ should be regarded as a cutoff function which allows us to get rid of the boundary.

Our claim is that, by choosing $k$ large enough, we have

$$
u(x)-u(y)-\Phi(x, y) \leq 0 \quad \forall x, y \in B .
$$

Indeed, suppose by contradiction that the function $w(x, y)=u(x)-u(y)-\Phi(x, y)$ is positive somewhere. Then, it should have a positive maximum taken at points $x$, $y$ inside $B$ and such that $x \neq y$. By a standard result in viscosity solutions theory (see e.g. [8]), for every $\varepsilon>0$ there exist matrices $X=X(\varepsilon), Y=Y(\varepsilon) \in \mathcal{S}_{N}$ such that

$$
\begin{aligned}
& \left(D_{x} \Phi(x, y), X\right) \in \bar{J}^{2,+} u(x), \quad\left(-D_{y} \Phi(x, y), Y\right) \in \bar{J}^{2,-} u(y), \\
& -\left(\frac{1}{\varepsilon}+\left\|D^{2} \Phi(x, y)\right\|\right) I_{2 N} \leq\left(\begin{array}{cc}
X & 0 \\
0 & -Y
\end{array}\right) \leq D^{2} \Phi(x, y)+\varepsilon\left(D^{2} \Phi(x, y)\right)^{2},
\end{aligned}
$$

where $\bar{J}^{2, \pm}$ denotes the closure of the second order super(sub)-jet.

Since $u$ is a viscosity subsolution of (3.1), this implies that

$$
-\operatorname{tr}(A(x) X)+\lambda u(x)+H\left(x, D_{x} \Phi\right) \leq 0,
$$

and, coupling with the condition of supersolution, that

$$
-\operatorname{tr}(A(x) X-A(y) Y)+\lambda(u(x)-u(y))+H\left(x, D_{x} \Phi\right)-H\left(y,-D_{y} \Phi\right) \leq 0 .
$$

In particular, for any $t>0$ we have

$$
\begin{gathered}
t \operatorname{tr}(A(x) X)-\operatorname{tr}((1+t) A(x) X-A(y) Y) \\
+\lambda(u(x)-u(y))+H\left(x, D_{x} \Phi\right)-H\left(y,-D_{y} \Phi\right) \leq 0,
\end{gathered}
$$

which yields, using (3.7) and since $u(x)-u(y)>k|x-y| \varphi>0$,

$t H\left(x, D_{x} \Phi\right) \leq-t \lambda u(x)+\operatorname{tr}((1+t) A(x) X-A(y) Y)+H\left(y,-D_{y} \Phi\right)-H\left(x, D_{x} \Phi\right)$. 
Now consider the nonnegative matrix

$$
\mathcal{A}_{t}=\left(\begin{array}{cc}
(1+t) \Sigma(x) \Sigma(x)^{T} & \sqrt{1+t} \Sigma(x) \Sigma(y)^{T} \\
\sqrt{1+t} \Sigma(y) \Sigma(x)^{T} & \Sigma(y) \Sigma(y)^{T}
\end{array}\right) ;
$$

multiplying the right-most inequality in (3.6) by $\mathcal{A}_{t}$ and taking traces yields

$$
\operatorname{tr}((1+t) A(x) X-A(y) Y) \leq \operatorname{tr}\left[\mathcal{A}_{t} D^{2} \Phi(x, y)\right]+\varepsilon \operatorname{tr}\left[\mathcal{A}_{t}\left(D^{2} \Phi(x, y)\right)^{2}\right] .
$$

Therefore we get from (3.9),

$$
\begin{aligned}
t H\left(x, D_{x} \Phi\right) \leq & -t \lambda u(x)+H\left(y,-D_{y} \Phi\right)-H\left(x, D_{x} \Phi\right) \\
& +\operatorname{tr}\left[\mathcal{A}_{t} D^{2} \Phi(x, y)\right]+\varepsilon \operatorname{tr}\left[\mathcal{A}_{t}\left(D^{2} \Phi(x, y)\right)^{2}\right],
\end{aligned}
$$

and finally, letting $\varepsilon$ tend to zero,

$$
t H\left(x, D_{x} \Phi\right) \leq-t \lambda u(x)+H\left(y,-D_{y} \Phi\right)-H\left(x, D_{x} \Phi\right)+\operatorname{tr}\left[\mathcal{A}_{t} D^{2} \Phi(x, y)\right]
$$

Now we have to compute the derivatives of $\Phi$. We get

$$
\begin{aligned}
D_{x} \Phi & =k \varphi \frac{x-y}{|x-y|}+k|x-y| D_{x} \varphi \\
& =\frac{k}{d(y)^{\gamma}}\left[\left(L+(1+\beta)\left(\frac{|x-y|}{d(x)}\right)^{\beta}\right) \frac{x-y}{|x-y|}-\beta\left(\frac{|x-y|}{d(x)}\right)^{\beta+1} D d(x)\right]
\end{aligned}
$$

and, similarly,

$$
\begin{aligned}
D_{y} \Phi=- & \frac{k}{d(y)^{\gamma}}\left[\left(L+(1+\beta)\left(\frac{|x-y|}{d(x)}\right)^{\beta}\right) \frac{x-y}{|x-y|}\right. \\
& \left.+\gamma \frac{|x-y|}{d(y)}\left(L+\left(\frac{|x-y|}{d(x)}\right)^{\beta}\right) \operatorname{Dd}(y)\right] .
\end{aligned}
$$

Henceforth, for shortness we will denote $\xi=\frac{|x-y|}{d(x)}$ and, for any vector $v \in \mathbb{R}^{N} \backslash\{0\}$, we set $\widehat{v}=\frac{v}{|v|}$. Moreover, we denote by $c$ several constants which may be different from line to line.

We notice that

$$
\begin{aligned}
\left|D_{x} \Phi\right|^{2}=\frac{k^{2}}{d(y)^{2 \gamma}}[ & \left(L+(1+\beta) \xi^{\beta}\right)^{2}+\beta^{2}|D d(x)|^{2} \xi^{2(\beta+1)} \\
& \left.-2 \beta \frac{D d(x) \cdot(x-y)}{d(x)} \xi^{\beta}\left(L+(1+\beta) \xi^{\beta}\right)\right] .
\end{aligned}
$$

Observe now that since the function $d(x)$ is nonnegative and concave in $B$, we have

$$
d(x) \geq D d(x) \cdot(x-y) \quad \forall x, y \in B ;
$$

hence we get

$$
\begin{aligned}
\left|D_{x} \Phi\right|^{2} & \geq \frac{k^{2}}{d(y)^{2 \gamma}}\left[\left(L+(1+\beta) \xi^{\beta}\right)^{2}+\beta^{2}|D d(x)|^{2} \xi^{2(\beta+1)}-2 \beta \xi^{\beta}\left(L+(1+\beta) \xi^{\beta}\right)\right] \\
& \geq \frac{k^{2}}{d(y)^{2 \gamma}}\left[\frac{1}{2}\left(L+(1+\beta) \xi^{\beta}\right)^{2}+\beta^{2}|D d(x)|^{2} \xi^{2(\beta+1)}-2 \beta^{2} \xi^{2 \beta}\right] .
\end{aligned}
$$


Moreover, by (3.4) we have $|D d(x)|=1$ if $d(x) \leq 1 / 2$ and $\xi=\frac{|x-y|}{d(x)} \leq 4$ if $d(x) \geq 1 / 2$. Thus we obtain

$$
\left|D_{x} \Phi\right|^{2} \geq \frac{k^{2}}{d(y)^{2 \gamma}}\left[\frac{1}{2}\left(L+(1+\beta) \xi^{\beta}\right)^{2}+\frac{\beta^{2}}{2} \xi^{2(\beta+1)}-c\right],
$$

where $c>0$ depends only on $\beta$. Here we choose $L$ sufficiently large such that

$$
\left|D_{x} \Phi\right|^{2} \geq c \frac{k^{2}}{d(y)^{2 \gamma}}\left[\left(L+(1+\beta) \xi^{\beta}\right)^{2}+\beta^{2} \xi^{2(\beta+1)}\right] .
$$

This will fix once for all the constant $L$. We conclude that there exists a constant $c>0$ such that

$$
\left|D_{x} \Phi\right| \geq c \frac{k}{d(y)^{\gamma}}\left(L+\xi^{\beta}\right)(1+\xi)=c k \varphi(1+\xi) .
$$

Let us now compute $D^{2} \Phi$. We have

$$
\begin{aligned}
D_{x x}^{2} \Phi= & \frac{k}{|x-y| d(y)^{\gamma}}\left[L+(1+\beta) \xi^{\beta}\right](I-\widehat{x-y} \otimes \widehat{x-y}) \\
& +\frac{k}{d(y)^{\gamma}} \beta(1+\beta) \frac{\xi^{\beta-1}}{d(x)} \widehat{x-y} \otimes \widehat{x-y} \\
& -\frac{k}{d(y)^{\gamma}} \beta(1+\beta) \frac{\xi^{\beta}}{d(x)}(\widehat{x-y} \otimes D d(x)+D d(x) \otimes \widehat{x-y}) \\
& +\frac{k}{d(y)^{\gamma}}\left[\beta(1+\beta) \frac{\xi^{\beta+1}}{d(x)} D d(x) \otimes D d(x)-\beta \xi^{\beta+1} D^{2} d(x)\right]
\end{aligned}
$$

and

$$
\begin{aligned}
D_{x y}^{2} \Phi= & -\frac{k}{|x-y| d(y)^{\gamma}}\left[L+(1+\beta) \xi^{\beta}\right](I-\widehat{x-y} \otimes \widehat{x-y}) \\
& -\frac{k}{d(y)^{\gamma}} \beta(1+\beta) \frac{\xi^{\beta-1}}{d(x)} \widehat{x-y} \otimes \widehat{x-y} \\
& +\frac{k}{d(y)^{\gamma}} \beta(1+\beta) \frac{\xi^{\beta}}{d(x)} D d(x) \otimes \widehat{x-y} \\
& -k \frac{\gamma}{d(y)^{\gamma+1}}\left[\left(L+(1+\beta) \xi^{\beta}\right) \widehat{x-y} \otimes D d(y)-\beta \xi^{\beta+1} \operatorname{Dd}(x) \otimes D d(y)\right]
\end{aligned}
$$

and

$$
\begin{aligned}
D_{y y}^{2} \Phi= & \frac{k}{|x-y| d(y)^{\gamma}}\left[L+(1+\beta) \xi^{\beta}\right](I-\widehat{x-y} \otimes \widehat{x-y}) \\
& +\frac{k}{d(y)^{\gamma}} \beta(1+\beta) \frac{\xi^{\beta-1}}{d(x)} \widehat{x-y} \otimes \widehat{x-y} \\
& +k \frac{\gamma}{d(y)^{\gamma+1}}\left(L+(1+\beta) \xi^{\beta}\right)(\widehat{x-y} \otimes D d(y)+D d(y) \otimes \widehat{x-y}) \\
& +k \gamma(\gamma+1) \frac{|x-y|}{d(y)^{\gamma+2}}\left(L+\xi^{\beta}\right) D d(y) \otimes D d(y) \\
& -k \gamma \frac{|x-y|}{d(y)^{\gamma+1}}\left(L+\xi^{\beta}\right) D^{2} d(y) .
\end{aligned}
$$


Therefore we can sum up $D^{2} \Phi$ as follows:

$$
\begin{gathered}
\frac{k}{d(y)^{\gamma}}\left\{\frac{\left(L+(1+\beta) \xi^{\beta}\right)}{|x-y|}\left(\begin{array}{cc}
B & -B \\
-B & B
\end{array}\right)+\frac{\beta(1+\beta) \xi^{\beta}}{|x-y|}\left(\begin{array}{cc}
T & -T \\
-T & T
\end{array}\right)\right. \\
+\frac{\beta(1+\beta) \xi^{\beta}}{d(x)}\left(\begin{array}{cc}
-\left(C+C^{T}\right) & C^{T} \\
C & O
\end{array}\right)+\frac{\gamma\left(L+(1+\beta) \xi^{\beta}\right)}{d(y)}\left(\begin{array}{cc}
O & -D \\
-D^{T} & D+D^{T}
\end{array}\right) \\
+\left(\begin{array}{cc}
\frac{\beta(\beta+1) \xi^{\beta+1}}{d(x)} D d(x) \otimes D d(x) & \frac{\beta \gamma \xi^{\beta+1}}{d(y)} D d(x) \otimes D d(y) \\
\frac{\beta \gamma \xi^{\beta+1}}{d(y)} D d(y) \otimes D d(x) & \frac{\gamma(\gamma+1)|x-y|}{d(y)^{2}}\left(L+\xi^{\beta}\right) D d(y) \otimes D d(y)
\end{array}\right) \\
\left.-\left(\begin{array}{cc}
\beta \xi^{\beta+1} D^{2} d(x) & O \\
O & \frac{\gamma|x-y|}{d(y)}\left(L+\xi^{\beta}\right) D^{2} d(y)
\end{array}\right)\right\},
\end{gathered}
$$

where $B, T, C$ and $D$ are the matrices defined respectively as $B=I-\widehat{x-y} \otimes \widehat{x-y}$, $T=\widehat{x-y} \otimes \widehat{x-y}, C=\widehat{x-y} \otimes D d(x), D=\widehat{x-y} \otimes D d(y)$. By recalling the definition of $\mathcal{A}_{t}$ in (3.10), we have

(3.15)

$$
\begin{aligned}
\operatorname{tr}\left[\mathcal{A}_{t} D^{2} \Phi\right. & (x, y)] \\
=\frac{k}{d(y)^{\gamma}}\{ & \frac{\left(L+(1+\beta) \xi^{\beta}\right)}{|x-y|} \operatorname{tr}\left[(\sqrt{1+t} \Sigma(x)-\Sigma(y))(\sqrt{1+t} \Sigma(x)-\Sigma(y))^{T} B\right] \\
& +\frac{\beta(1+\beta) \xi^{\beta}}{|x-y|} \operatorname{tr}\left[(\sqrt{1+t} \Sigma(x)-\Sigma(y))(\sqrt{1+t} \Sigma(x)-\Sigma(y))^{T} T\right] \\
& -2 \frac{\beta(1+\beta) \xi^{\beta}}{d(x)} \sqrt{1+t} \operatorname{tr}\left[\Sigma(x)(\sqrt{1+t} \Sigma(x)-\Sigma(y))^{T} C\right] \\
& -2 \frac{\gamma\left(L+(1+\beta) \xi^{\beta}\right)}{d(y)} \operatorname{tr}\left[\Sigma(y)(\sqrt{1+t} \Sigma(x)-\Sigma(y))^{T} D\right] \\
& +\frac{\beta(1+\beta) \xi^{\beta+1}}{d(x)}(1+t)\left|\Sigma(x)^{T} D d(x)\right|^{2} \\
& +\frac{\gamma(\gamma+1)|x-y|\left(L+\xi^{\beta}\right)}{d(y)^{2}}\left|\Sigma(y)^{T} D d(y)\right|^{2} \\
& +2 \frac{\beta \gamma \xi^{\beta+1}}{d(y)} \sqrt{1+t} \Sigma(x)^{T} D d(x) \cdot \Sigma(y)^{T} D d(y) \\
& \left.-\beta \xi^{\beta+1}(1+t) \operatorname{tr}\left(A(x) D^{2} d(x)\right)-\frac{\gamma|x-y|\left(L+\xi^{\beta}\right)}{d(y)} \operatorname{tr}\left(A(y) D^{2} d(y)\right)\right\} .
\end{aligned}
$$

In order to estimate the first terms in the right hand side, observe that, by the Lipschitz continuity of $\Sigma$, we have ( $\left\|\Sigma_{x}\right\|$ being the Lipschitz constant)

$$
\begin{aligned}
\operatorname{tr} & {\left[(\sqrt{1+t} \Sigma(x)-\Sigma(y))(\sqrt{1+t} \Sigma(x)-\Sigma(y))^{T} B\right] } \\
& \leq[(\sqrt{1+t}-1)\|\Sigma\|+\|\Sigma(x)-\Sigma(y)\|]^{2} \\
& \leq 2\left(t^{2}\|\Sigma\|^{2}+\left\|\Sigma_{x}\right\|^{2}|x-y|^{2}\right) .
\end{aligned}
$$


Similarly, we have

$$
\begin{aligned}
& \sqrt{1+t} \operatorname{tr}\left[\Sigma(x)(\sqrt{1+t} \Sigma(x)-\Sigma(y))^{T} C\right] \\
& =t \operatorname{tr}\left[\Sigma(x) \Sigma(x)^{T} C\right]+\operatorname{tr}\left[\Sigma(x)(\Sigma(x)-\sqrt{1+t} \Sigma(y))^{T} C\right] \\
& \quad \leq 2 t\|\Sigma\|^{2}+\|\Sigma\|\left\|\Sigma_{x}\right\||x-y|, \\
& \operatorname{tr}\left[\Sigma(y)(\sqrt{1+t} \Sigma(x)-\Sigma(y))^{T} D\right] \leq t\|\Sigma\|^{2}+\|\Sigma\|\left\|\Sigma_{x}\right\||x-y| .
\end{aligned}
$$

We obtain then from (3.15),

$$
\begin{aligned}
\operatorname{tr}\left[\mathcal{A}_{t} D^{2} \Phi(x, y)\right] \leq & \frac{c k}{d(y)^{\gamma}}\left\{\frac{\left(L+\xi^{\beta}\right)}{|x-y|}\left(t^{2}\|\Sigma\|^{2}+|x-y|^{2}\left\|\Sigma_{x}\right\|^{2}\right)\right. \\
& +\left(\frac{\xi^{\beta}}{d(x)}+\frac{\left(1+\xi^{\beta}\right)}{d(y)}\right)\left(t\|\Sigma\|^{2}+|x-y|\|\Sigma\|\left\|\Sigma_{x}\right\|\right) \\
& +\xi^{\beta+1}\left(\frac{1}{d(x)}+\frac{1}{d(y)}\right) t\|\Sigma\|^{2} \\
& \left.+\|\Sigma\|^{2}\left(\frac{\xi^{\beta+1}}{d(x)}+\frac{\xi^{\beta+1}}{d(y)}+\frac{\left(1+\xi^{\beta}\right)|x-y|}{d(y)^{2}}\right)\right\} .
\end{aligned}
$$

Observe now that

$$
\left\{\begin{array}{l}
\xi=\frac{|x-y|}{d(x)} \leq 1 \Rightarrow d(y) \leq 2 d(x) \\
\xi \geq 1 \Rightarrow d(y) \leq 2|x-y|
\end{array}\right.
$$

hence

$$
\frac{\xi^{\beta}}{d(x)} \leq 2 \frac{\left(1+\xi^{\beta+1}\right)}{d(y)}
$$

Moreover, by definition of $\xi$,

$$
\left(\frac{\xi^{\beta+1}}{d(x)}+\frac{\xi^{\beta+1}}{d(y)}+\frac{\left(1+\xi^{\beta}\right)|x-y|}{d(y)^{2}}\right)=|x-y|\left(\frac{\xi^{\beta}}{d(x)^{2}}+\frac{\xi^{\beta}}{d(y) d(x)}+\frac{\left(1+\xi^{\beta}\right)}{d(y)^{2}}\right)
$$

and by using (3.17) again we get

$$
\left(\frac{\xi^{\beta+1}}{d(x)}+\frac{\xi^{\beta+1}}{d(y)}+\frac{\left(1+\xi^{\beta}\right)|x-y|}{d(y)^{2}}\right) \leq c|x-y| \frac{\left(1+\xi^{\beta+2}\right)}{d(y)^{2}} .
$$

Therefore (3.16) implies

$$
\begin{aligned}
& \operatorname{tr}\left[\mathcal{A}_{t} D^{2} \Phi(x, y)\right] \\
& \leq \frac{c k}{d(y)^{\gamma}}\left\{\frac{\left(L+\xi^{\beta}\right)}{|x-y|}\left(t^{2}\|\Sigma\|^{2}+|x-y|^{2}\left\|\Sigma_{x}\right\|^{2}\right)+t \frac{\left(1+\xi^{\beta+2}\right)}{d(y)}\|\Sigma\|^{2}\right. \\
& \left.\quad+\frac{\left(1+\xi^{\beta+1}\right)}{d(y)}|x-y|\|\Sigma\|\left\|\Sigma_{x}\right\|+\frac{\left(1+\xi^{\beta+2}\right)}{d(y)^{2}}|x-y|\|\Sigma\|^{2}\right\} .
\end{aligned}
$$

From (3.11) it then follows

$$
\begin{aligned}
& t H\left(x, D_{x} \Phi\right) \leq-t \lambda u(x)+H\left(y,-D_{y} \Phi\right)-H\left(x, D_{x} \Phi\right) \\
& \quad+\frac{c k}{d(y)^{\gamma}}\left\{\frac{\left(L+\xi^{\beta}\right)}{|x-y|}\left(t^{2}\|\Sigma\|^{2}+|x-y|^{2}\left\|\Sigma_{x}\right\|^{2}\right)+t \frac{\left(1+\xi^{\beta+2}\right)}{d(y)}\|\Sigma\|^{2}\right. \\
& \left.\quad+\frac{\left(1+\xi^{\beta+1}\right)}{d(y)}|x-y|\|\Sigma\|\left\|\Sigma_{x}\right\|+\frac{\left(1+\xi^{\beta+2}\right)}{d(y)^{2}}|x-y|\|\Sigma\|^{2}\right\} .
\end{aligned}
$$


By the growth condition (3.3) on $H$ we deduce

$$
\begin{aligned}
t \gamma_{0}\left|D_{x} \Phi\right|^{p} \leq & t(f(x)-\lambda u(x))+H\left(y,-D_{y} \Phi\right)-H\left(x, D_{x} \Phi\right) \\
& +\frac{c k}{d(y)^{\gamma}}\left\{\frac{\left(L+\xi^{\beta}\right)}{|x-y|}\left(t^{2}\|\Sigma\|^{2}+|x-y|^{2}\left\|\Sigma_{x}\right\|^{2}\right)+t \frac{\left(1+\xi^{\beta+2}\right)}{d(y)}\|\Sigma\|^{2}\right. \\
& \left.+\frac{\left(1+\xi^{\beta+1}\right)}{d(y)}|x-y|\|\Sigma\|\left\|\Sigma_{x}\right\|+\frac{\left(1+\xi^{\beta+2}\right)}{d(y)^{2}}|x-y|\|\Sigma\|^{2}\right\} .
\end{aligned}
$$

Observe now that choosing $\gamma \geq \frac{1}{p-1}$ and $\beta \geq \frac{2-p}{p-1}$ gives $\gamma+1 \leq \gamma p$ and $\beta+2 \leq$ $(\beta+1) p$; hence, by estimate (3.14),

$$
\left|D_{x} \Phi\right|^{p} \geq c k^{p} \frac{\left(1+\xi^{\beta+1}\right)^{p}}{d(y)^{\gamma p}} \geq c k^{p} \frac{\left(1+\xi^{\beta+2}\right)}{d(y)^{\gamma+1}} .
$$

Therefore, as soon as $k^{p-1} \gg \frac{1}{\gamma_{0}}\|\Sigma\|^{2}$, we get

$$
\begin{aligned}
& t \frac{\gamma_{0}}{2}\left|D_{x} \Phi\right|^{p}-c k t^{2}\|\Sigma\|^{2} \frac{\left(L+\xi^{\beta}\right)}{|x-y| d(y)^{\gamma}} \\
& \leq \quad t(f(x)-\lambda u(x))+H\left(y,-D_{y} \Phi\right)-H\left(x, D_{x} \Phi\right) \\
& \quad+c \frac{k|x-y|}{d(y)^{\gamma}}\left[\left(1+\xi^{\beta}\right)\left\|\Sigma_{x}\right\|^{2}+\frac{\left(1+\xi^{\beta+1}\right)}{d(y)}\|\Sigma\|\left\|\Sigma_{x}\right\|+\frac{\left(1+\xi^{\beta+2}\right)}{d(y)^{2}}\|\Sigma\|^{2}\right] .
\end{aligned}
$$

Here we make the (optimal) choice of $t$ in order to maximize the left hand side. This yields

$$
t=c \frac{\left|D_{x} \Phi\right|^{p}}{k\|\Sigma\|^{2} \frac{\left(L+\xi^{\beta}\right)}{|x-y| d(y)^{\gamma}}}=c \frac{\left|D_{x} \Phi\right|^{p}}{k\|\Sigma\|^{2}} \frac{|x-y|}{\varphi}
$$

by recalling the definition of the cutoff function $\varphi$ in (3.5). We then obtain

$$
\begin{aligned}
& \frac{\left|D_{x} \Phi\right|^{2 p}}{k\|\Sigma\|^{2}} \frac{|x-y|}{\varphi} \\
& \leq c\left\{\frac{\left|D_{x} \Phi\right|^{p}}{k\|\Sigma\|^{2}} \frac{|x-y|}{\varphi}\left\|(f-\lambda u)^{+}\right\|+H\left(y,-D_{y} \Phi\right)-H\left(x, D_{x} \Phi\right)\right. \\
& \left.\quad+k|x-y|\left[\varphi\left\|\Sigma_{x}\right\|^{2}+\frac{\left(1+\xi^{\beta+1}\right)}{d(y)^{\gamma+1}}\|\Sigma\|\left\|\Sigma_{x}\right\|+\frac{\left(1+\xi^{\beta+2}\right)}{d(y)^{\gamma+2}}\|\Sigma\|^{2}\right]\right\} ;
\end{aligned}
$$

hence

(3.20)

$$
\begin{aligned}
\left|D_{x} \Phi\right|^{2 p} \leq c & \left\{\left|D_{x} \Phi\right|^{p}\left\|(f-\lambda u)^{+}\right\|+k\|\Sigma\|^{2} \frac{\varphi}{|x-y|}\left[H\left(y,-D_{y} \Phi\right)-H\left(x, D_{x} \Phi\right)\right]\right. \\
& \left.+k^{2} \varphi\|\Sigma\|^{2}\left[\varphi\left\|\Sigma_{x}\right\|^{2}+\frac{\left(1+\xi^{\beta+2}\right)}{d(y)^{\gamma+2}}\left(\|\Sigma\|^{2}+\left\|\Sigma_{x}\right\|^{2}\right)\right]\right\} .
\end{aligned}
$$

Moreover, by assumption (3.3) on $H$, it follows that

$$
\begin{aligned}
H\left(y,-D_{y} \Phi\right)-H\left(x, D_{x} \Phi\right) \leq & \gamma_{1}\left(\left|D_{x} \Phi\right|^{p-1}+\left|D_{y} \Phi\right|^{p-1}\right)\left|D_{x} \Phi+D_{y} \Phi\right| \\
& +\gamma_{2}|x-y|\left(\left\|g_{1}\right\|+\left\|g_{2}\right\|\left|D_{x} \Phi\right|^{p}\right) .
\end{aligned}
$$


By recalling (3.12) and (3.13) and by again using (3.17), we have

$$
\begin{aligned}
& \left(\left|D_{x} \Phi\right|^{p-1}+\left|D_{y} \Phi\right|^{p-1}\right)\left|D_{x} \Phi+D_{y} \Phi\right| \\
& \leq c k^{p} \frac{\left(1+\xi^{\beta}\right)^{p}}{d(y)^{\gamma p}}\left(1+\frac{|x-y|}{d(x)} \vee \frac{|x-y|}{d(y)}\right)^{p-1} \frac{|x-y|}{d(x)} \vee \frac{|x-y|}{d(y)} \\
& \leq c k^{p} \frac{\left(1+\xi^{\beta+1}\right)^{p}}{d(y)^{\gamma p+p}}|x-y|,
\end{aligned}
$$

so that

$$
\begin{aligned}
\left|D_{x} \Phi\right|^{2 p} \leq c\{ & \left|D_{x} \Phi\right|^{p}\left\|(f-\lambda u)^{+}\right\|+k\|\Sigma\|^{2} \varphi\left(\left\|g_{1}\right\|+\left\|g_{2}\right\|\left|D_{x} \Phi\right|^{p}\right) \\
& +k^{p+1}\|\Sigma\|^{2} \varphi \frac{\left(1+\xi^{\beta+1}\right)^{p}}{d(y)^{\gamma p+p}} \\
& \left.+k^{2} \varphi\|\Sigma\|^{2}\left[\varphi\left\|\Sigma_{x}\right\|^{2}+\frac{\left(1+\xi^{\beta+2}\right)}{d(y)^{\gamma+2}}\left(\|\Sigma\|^{2}+\left\|\Sigma_{x}\right\|^{2}\right)\right]\right\} .
\end{aligned}
$$

Now we choose $\gamma=\frac{p}{p-1}$ and from (3.14) it follows that

$$
k^{p+1} \varphi \frac{\left(1+\xi^{\beta+1}\right)^{p}}{d(y)^{\gamma p+p}} \leq c k \frac{\varphi}{d(y)^{p}}\left|D_{x} \Phi\right|^{p} \leq c k \varphi^{p}\left|D_{x} \Phi\right|^{p} \leq \frac{c}{k^{p-1}}\left|D_{x} \Phi\right|^{2 p} .
$$

Furthermore, since $2(\gamma+1) \leq 2 \gamma p$ and $\beta+2 \leq(\beta+1) p$, we also have

$k^{2} \varphi \frac{\left(1+\xi^{\beta+2}\right)}{d(y)^{\gamma+2}} \leq c k^{2} \frac{\left(1+\xi^{\beta}\right)}{d(y)^{\gamma}} \frac{\left(1+\xi^{\beta+1}\right)^{p}}{d(y)^{\gamma+2}} \leq c k^{2} \frac{\left(1+\xi^{\beta+1}\right)^{2 p}}{d(y)^{2 \gamma p}} \leq \frac{c}{k^{2(p-1)}}\left|D_{x} \Phi\right|^{2 p}$.

Then, we deduce that

$$
\begin{aligned}
\left|D_{x} \Phi\right|^{2 p} \leq c & \left\{\left|D_{x} \Phi\right|^{p}\left\|(f-\lambda u)^{+}\right\|+\|\Sigma\|^{2}\left[\left|D_{x} \Phi\right|\left(\left\|g_{1}\right\|+\left\|g_{2}\right\|\left|D_{x} \Phi\right|^{p}\right)\right.\right. \\
& \left.\left.+\frac{\left|D_{x} \Phi\right|^{2 p}}{k^{p-1}}+\left\|\Sigma_{x}\right\|^{2}\left|D_{x} \Phi\right|^{2}+\frac{\left(\|\Sigma\|^{2}+\left\|\Sigma_{x}\right\|^{2}\right)}{k^{2(p-1)}}\left|D_{x} \Phi\right|^{2 p}\right]\right\} .
\end{aligned}
$$

Since $p>1$, for $k$ large we get a contradiction.

The above argument shows that there exists a positive constant $M$ depending on $p,\|\Sigma\|_{L^{\infty}(B)},\left\|\Sigma_{x}\right\|_{L^{\infty}(B)},\left\|(f-\lambda u)^{+}\right\|_{L^{\infty}(B)},\left\|g_{1}\right\|_{L^{\infty}(B)},\left\|g_{2}\right\|_{L^{\infty}(B)}, \gamma_{0}, \gamma_{1}, \gamma_{2}$ such that

$$
u(x)-u(y) \leq M \frac{|x-y|}{d_{\partial B}(y)^{\gamma}}\left[1+\left(\frac{|x-y|}{d_{\partial B}(x)}\right)^{\beta}\right] \quad \forall x, y \in B .
$$

By reversing the role of $x$ and $y$ we get

$$
|u(x)-u(y)| \leq M \frac{|x-y|}{\left(d_{\partial B}(x) \wedge d_{\partial B}(y)\right)^{\gamma}}\left[1+\left(\frac{|x-y|}{d_{\partial B}(x) \wedge d_{\partial B}(y)}\right)^{\beta}\right] \quad \forall x, y \in B,
$$

so that $u$ is locally Lipschitz continuous in $B$ and satisfies $|D u(x)| \leq \frac{M}{d_{\partial B}(x)^{\gamma}}$ for almost every $x \in B$. On the other hand, the exponent $\gamma=\frac{p}{p-1}$ found in the 
previous proof is not optimal: in order to recover the right exponent it is enough to make a suitable rescaling.

To this purpose, let $x_{0} \in \Omega$ be a differentiability point for $u$ and let $r=\frac{d_{\partial \Omega}\left(x_{0}\right)}{2}$. Consider the rescaled function

$$
v(x)=r^{\frac{2-p}{p-1}} u\left(x_{0}+r x\right)
$$

defined for $x \in B=B_{1}(0)$. An easy computation shows that $v$ is a viscosity solution of the equation

$$
-\operatorname{tr}\left(A_{r}(x) D^{2} v\right)+r^{2} \lambda v+H_{r}(x, D v)=0, \quad x \in B,
$$

where $A_{r}(x)=A\left(x_{0}+r x\right)$ and $H_{r}(x, \xi)=r^{\frac{p}{p-1}} H\left(x_{0}+r x, r^{-\frac{1}{p-1}} \xi\right)$. Note that $H_{r}$ satisfies (3.3) with the same constants but replacing $f$ with $f_{r}(x)=r^{\frac{p}{p-1}} f\left(x_{0}+r x\right)$ and $g_{1}, g_{2}$ with $g_{1, r}(x)=r^{\frac{2 p-1}{p-1}} g_{1}\left(x_{0}+r x\right)$ and $g_{2, r}(x)=r g_{2}\left(x_{0}+r x\right)$, respectively. Now, since $r=\frac{d_{\partial \Omega}\left(x_{0}\right)}{2}$, we have

$$
r \leq d_{\partial \Omega}\left(x_{0}+r x\right) \leq 3 r
$$

hence

$$
\begin{aligned}
\left\|\left(f_{r}-\lambda r^{2} v\right)^{+}\right\|_{L^{\infty}(B)} & =\left\|r^{\frac{p}{p-1}}\left(f\left(x_{0}+r x\right)-\lambda u\left(x_{0}+r x\right)\right)^{+}\right\|_{L^{\infty}(B)} \\
& \leq\left\|d_{\partial \Omega}^{\frac{p}{p-1}}(f-\lambda u)^{+}\right\|_{L^{\infty}(\Omega)} .
\end{aligned}
$$

Similarly we have

$$
\left\|g_{1, r}\right\|_{L^{\infty}(B)} \leq\left\|d_{\partial \Omega}^{\frac{2 p-1}{p-1}} g_{1}\right\|_{L^{\infty}(\Omega)}, \quad\left\|g_{2, r}\right\|_{L^{\infty}(B)} \leq\left\|d_{\partial \Omega} g_{2}\right\|_{L^{\infty}(\Omega)} .
$$

By the result obtained in the ball we have (note that 0 is a differentiability point for $v$ )

$$
|D v(0)| \leq M,
$$

with a constant $M>0$ depending on $p,\|\Sigma\|_{L^{\infty}},\left\|\Sigma_{x}\right\|_{L^{\infty}},\left\|d_{\partial \Omega}^{\frac{p}{p-1}}(f-\lambda u)^{+}\right\|_{L^{\infty}}$, $\left\|d_{\partial \Omega}^{\frac{2 p-1}{p-1}} g_{1}\right\|_{L^{\infty}(\Omega)},\left\|d_{\partial \Omega} g_{2}\right\|_{L^{\infty}(\Omega)}$, and on the constants $\gamma_{0}, \gamma_{1}$ and $\gamma_{2}$.

Hence we conclude that

$$
\left|D u\left(x_{0}\right)\right| \leq M r^{-\frac{1}{p-1}}=\frac{K}{d_{\partial \Omega}\left(x_{0}\right)^{\frac{1}{p-1}}} .
$$

Remark 3.2. The above method can be applied to different Hamiltonians $H(x, \xi)$ and, correspondingly, different adapted choices of cutoff functions $\varphi$. Anyway, this approach requires the coercivity of the Hamiltonian (a somehow necessary ingredient for having estimates in the case of degenerate ellipticity; see also [14]). Indeed, if we rephrase our proof for general functions $H$, the optimal choice of the parameter $t$ in (3.19) would read as

$$
t=c \frac{H\left(x, D_{x}(\Phi)\right)}{k\|\Sigma\|^{2}} \frac{|x-y|}{\varphi},
$$

where $\varphi$ is the cutoff function. Observe that by construction we have $\left|D_{x} \Phi\right| \rightarrow \infty$; hence we need $H(x, p)$ to be positive as $|p| \rightarrow \infty$ in order that $t>0$. Moreover, 
recalling in the previous proof the definition of $\varphi=\frac{(L+\xi)^{\beta}}{d(y)^{\gamma}}$ and estimate (3.14), the inequality (3.20) could have been rephrased in the more abstract form

$$
\begin{aligned}
H^{2}\left(x, D_{x} \Phi\right) & \leq c\left\|\lambda u^{-}\right\|_{L^{\infty}(\Omega)}+c\|\Sigma\|^{2}\left\{\frac{\left|D_{x} \Phi\right|}{|x-y|}\left[H\left(y,-D_{y} \Phi\right)-H\left(x, D_{x} \Phi\right)\right]\right. \\
& \left.+\left\|\Sigma_{x}\right\|^{2}\left|D_{x} \Phi\right|^{2}+\left(\|\Sigma\|^{2}+\left\|\Sigma_{x}\right\|^{2}\right)\left|D_{x} \Phi\right|^{2} \varphi^{q}\right\}
\end{aligned}
$$

where $q=\max \left(\frac{1}{\beta}, \frac{1}{\gamma}\right)$.

From a similar estimate, one can conclude the proof provided some suitable coercivity condition on $H(x, p)$ holds as $|p| \rightarrow \infty$. In particular, let us note that if we disregard the localization function and take $\varphi=1$, then $D_{x} \Phi=-D_{y} \Phi$ and it would be enough to assume, for some constant $c_{0}$ :

$$
\liminf _{|p| \rightarrow \infty} \frac{c_{0} H^{2}(x, p)-\|\Sigma\|^{2}|p| \frac{[H(y, p)-H(x, p)]}{|x-y|}}{|p|^{2}}>2\|\Sigma\|^{2}\left(\|\Sigma\|^{2}+\left\|\Sigma_{x}\right\|^{2}\right) .
$$

This assumption (which, if one really takes $\varphi=1$, could be further refined with only $\|\Sigma\|^{2}\left\|\Sigma_{x}\right\|^{2}$ in the right hand side) is analogous to the one used in [14 to get global Lipschitz estimates for solutions in the whole space. This shows that our approach can replace, in some situations, the classical Bernstein method of differentiating the equation. On the other hand, the explicit superlinear character asked in assumption (3.3) was needed to handle the difficulties of the localization argument through the explicit construction of the cutoff function $\varphi$.

\section{REFERENCES}

[1] M. Bardi, I. Capuzzo Dolcetta, Optimal Control and Viscosity Solutions of Hamilton-JacobiBellman Equations, Systems \& Control: Foundations \& Applications, Birkhäuser Boston, Inc., Boston, MA, 1997. MR.1484411 (99e:49001)

[2] G. Barles, Solutions de viscosité des équations de Hamilton-Jacobi, Mathématiques \& Applications 17, Springer-Verlag, Paris, 1994. MR.1613876 (2000b:49054)

[3] G. Barles, A weak Bernstein method for fully nonlinear elliptic equations, Differential Integral Equations 4 (1991), 241-262. MR1081182(91k:35087)

[4] G. Barles, F. Da Lio, On the generalized Dirichlet problem for viscous Hamilton-Jacobi equations, J. Math. Pures Appl. (9) 83 (2004), no. 1, 53-75. MR2023054 (2005e:35107)

[5] G. Barles, B. Perthame, Comparison principle for Dirichlet-type Hamilton-Jacobi equations and singular perturbations of degenerated elliptic equations, Appl. Math. Optim. 21 (1990), no. 1, 21-44. MR 1014943 (90m:35017)

[6] L. Caffarelli, M. G. Crandall, M. Kocan, A. Swiech, On viscosity solutions of fully nonlinear equations with measurable ingredients, Comm. Pure Appl. Math. 49 (1996), no. 4, 365-397. MR1376656 (97a:35051)

[7] F. H. Clarke, Optimization and nonsmooth analysis. Canadian Mathematical Society Series of Monographs and Advanced Texts. A Wiley-Interscience Publication, John Wiley \& Sons, Inc., New York, 1983. MR.709590 (85m:49002)

[8] M. Crandall, H. Ishii, P.L. Lions, User's guide to viscosity solutions of second order partial differential equations, Bulletin of the American Mathematical Society (1992), Volume 27, Number 1. MR.1118699 (92j:35050)

[9] W. H. Fleming, M. H. Soner, Controlled Markov Processes and Viscosity Solutions, Applications of Mathematics 25, Springer-Verlag, 1991. MR2179357 (2006e:93002)

[10] J.M. Lasry, P.L. Lions, Nonlinear elliptic equations with singular boundary conditions and stochastic control with state constraints. I. The model problem, Math. Ann. 283 (1989), no. 4, 583-630. MR 990591 (90f:35072)

[11] P.L. Lions, Résolution de problèmes elliptiques quasilinéaires, (French) Arch. Rational Mech. Anal. 74 (1980), no. 4, 335-353. MR588033 (82a:35034) 
[12] P.L. Lions, Generalized solutions of Hamilton-Jacobi equations, Research Notes in Mathematics 69, Pitman (Advanced Publishing Program), Boston, Mass.-London, 1982. MR667669 (84a:49038)

[13] P.L. Lions, Quelques remarques sur les problèmes elliptiques quasilinéaires du second ordre, (French) J. Analyse Math. 45 (1985), 234-254. MR833413 (87f:35088)

[14] P.L. Lions, P.E. Souganidis, Homogenization of degenerate second-order PDE in periodic and almost periodic environments and applications, Ann. Inst. H. Poincaré Anal. Non Linéaire 22 (2005), no. 5, 667-677. MR2171996 (2006d:35019)

[15] R.T. Rockafellar, Clarke's tangent cones and the boundaries of closed sets in $\mathbb{R}^{n}$, Nonlin. Anal. T.M.A. 3 (1979), no. 1, 145-154. MR.520481(80d:49032)

Dipartimento di Matematica, Sapienza Università di Roma, P.le A. Moro 2, 00185 Roma, ITALY

E-mail address: capuzzo@mat.uniroma1.it

Dipartimento di Matematica, Sapienza Università di Roma, P.le A. Moro 2, 00185 ROMA, ITALY

E-mail address: leoni@mat.uniroma1.it

Dipartimento di Matematica, Università di Roma tor Vergata, Via della Ricerca Scientifica 1, 00133 Roma, Italy

E-mail address: porretta@mat.uniroma2.it 